\title{
Asic developments for radiation imaging applications: The medipix and timepix family
}

\author{
Rafael Ballabriga, Michael Campbell *, Xavier Llopart
}

CERN, 1211 Geneva 23, Switzerland

\section{A R T I C L E I N F O}

\section{Keywords:}

Hybrid pixel detector

Medipix

Timepix

X-ray imaging

Particle detection

Particle tracking

\begin{abstract}
A $\quad$ B $\quad S \quad T \quad R \quad A \quad C$ T
Hybrid pixel detectors were developed to meet the requirements for tracking in the inner layers at the LHC experiments. With low input capacitance per channel (10-100 fF) it is relatively straightforward to design pulse processing readout electronics with input referred noise of $\sim 100$ e-rms and pulse shaping times consistent with tagging of events to a single LHC bunch crossing providing clean 'images' of the ionising tracks generated. In the Medipix Collaborations the same concept has been adapted to provide practically noise hit free imaging in a wide range of applications. This paper reports on the development of three generations of readout ASICs. Two distinctive streams of development can be identified: the Medipix ASICs which integrate data from multiple hits on a pixel and provide the images in the form of frames and the Timepix ASICs who aim to send as much information about individual interactions as possible off-chip for further processing. One outstanding circumstance in the use of these devices has been their numerous successful applications, thanks to a large and active community of developers and users. That process has even permitted new developments for detectors for High Energy Physics. This paper reviews the ASICs themselves and details some of the many applications.
\end{abstract}

(C) 2017 The Authors. Published by Elsevier B.V. This is an open access article under the CC BY license (http://creativecommons.org/licenses/by/4.0/).

\section{Contents}

1. Introduction

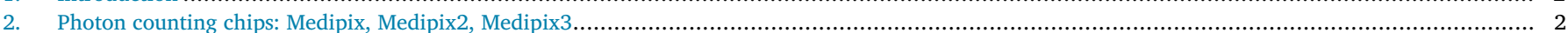

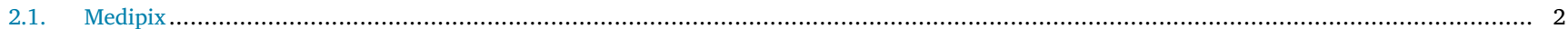

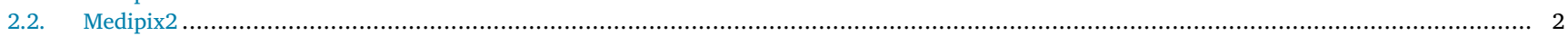

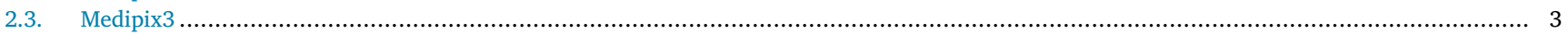

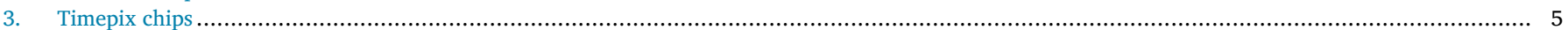

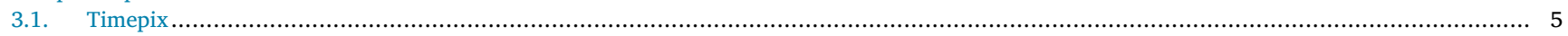

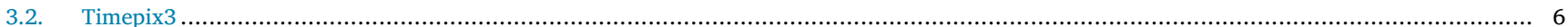

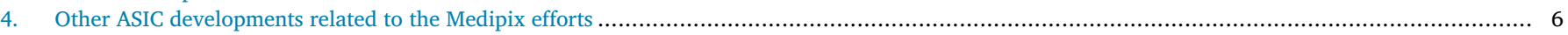

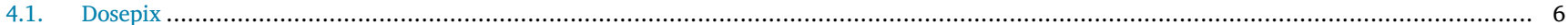

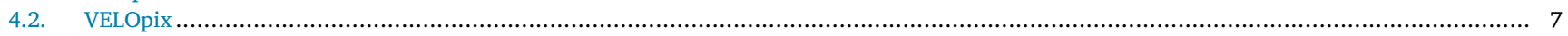

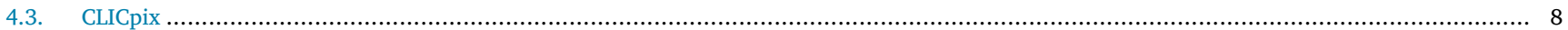

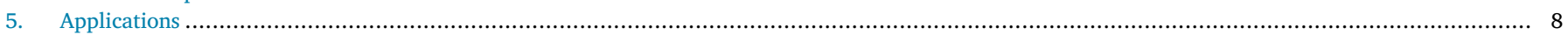

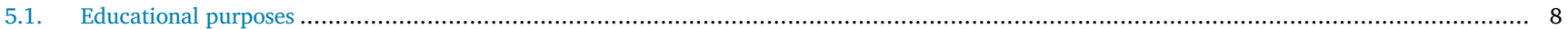

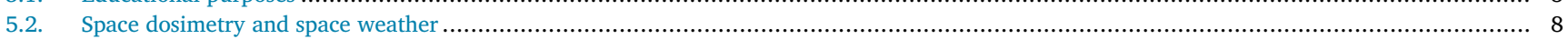

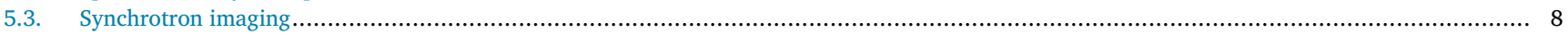

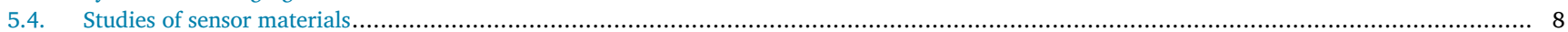

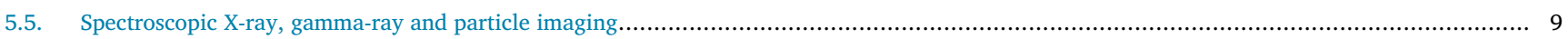

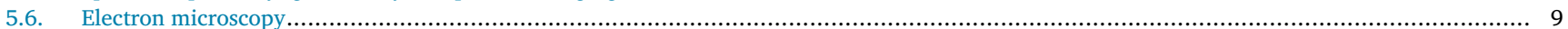

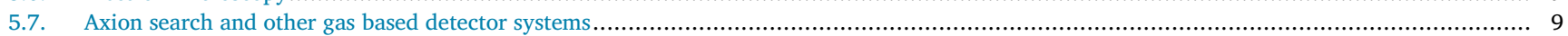

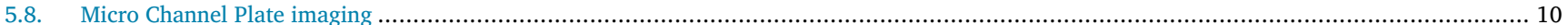

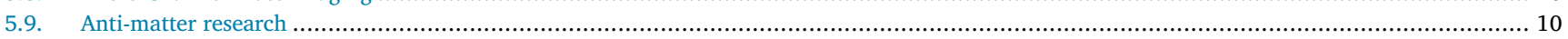

\footnotetext{
* Corresponding author.

E-mail address: michael.campbell@cern.ch (M. Campbell).
} 


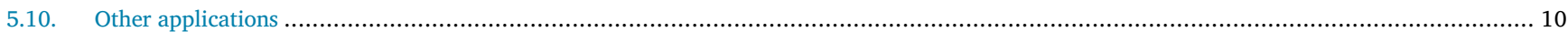

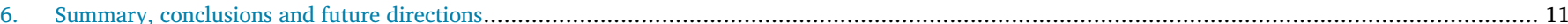

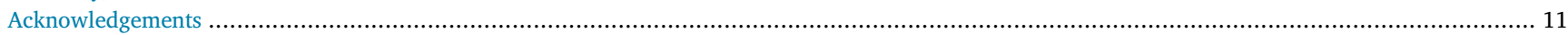

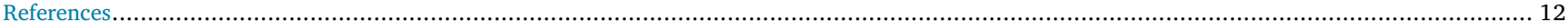

\section{Introduction}

Hybrid pixel detectors with on-pixel pulse processing were developed first for use at the LHC and a history of the early developments in what became commonly referred to as, "pixel detectors" can be found in [1]. These replaced strip detectors in the inner tracking layers of the vertex detectors where track density was so high that a 2-D geometry was required to permit correct pattern recognition. Moreover, because of the close proximity of sensor and readout electronics the input capacitance of a channel is low (10-100 fF) and input referred noise levels of around $100 \mathrm{e}^{-}$rms are relatively easy to achieve even at shaping times consistent with proper allocation of a hit to one LHC bunch crossing (25 ns). As the operating threshold is set at around 1000$2000 \mathrm{e}^{-}$the "images" of bunch crossing are essentially "noise hit free". Because of readout bandwidth restrictions only selected or "triggered" events are read out from such detectors. Radiation imaging detectors on the other hand usually accumulate data on the detector prior to readout. In the early days of the first Medipix Collaboration we sought simply to generate "noise free" images on chip by accumulating hit counts on each pixel. Over the years this concept has extended to extracting as much information as possible about the incoming ionising particles and sending it off chip.

This paper reports firstly on our imaging chip devices Medipix, Medipix2 and Medipix3. In these devices, single hits are processed either on-pixel or within a neighbourhood of pixels and counts are accumulated locally on-pixel prior to frame-based readout. In a second part, we describe the Timepix chips where as much data as possible is generated locally on-pixel and then sent off-chip for processing. These chips can record particle arrival time and/or the energy deposited per pixel. A third section describes chips which have been directly derived from the Medipix and Timepix family and finally in the 4th section a number of applications to which these chips have been applied are described.

\section{Photon counting chips: Medipix, Medipix2, Medipix3}

The first Medipix chip was submitted to fabrication in 1997. The pixel front-end of that chip was derived directly from the Omega3/LHC1 chip [2] which was developed in the context of the R and D for the then future LHC machine. The Omega3 chip, as the name hints, was already the 3rd generation of pixel detector chips developed for high energy physics applications; pilot biomedical imaging studies were reported with this photon counting detector as early as 20 years ago $[3,4]$. The first Medipix chip [5] uses a $1 \mu \mathrm{m}$ minimum feature size. Today we are designing with a minimum feature size of $65 \mathrm{~nm}$.

\subsection{Medipix}

The first Medipix chip consisted of a matrix of $64 \times 64$ square cells on a pitch of $170 \mu \mathrm{m}$. An externally applied shutter places the chip in either acquisition mode or readout mode. Details of the chip can be found in [6]. A schematic diagram of the Medipix pixel cell is shown in Fig. 1. A front-end preamplifier integrates an incoming charge pulse to a $\sim 3.5 \mathrm{fF}$ feedback capacitor providing a gain of $\sim 30 \mathrm{mV} / \mathrm{ke}^{-}$. Detector leakage current is compensated for by subtracting the current measured in a dummy cell at the bottom of each column from each preamp input.
This means that the sensor matrix has $64 \times 65$ cells but that only $64 \times$ 64 cells are read out. This is a significant limitation when sensors with non-uniform leakage current are connected to the chip. A discriminator is connected to the output of each preamplifier. A global threshold is applied using and external current source but the local threshold can be tuned using a programmable 3-bit register in each pixel. The range of the 3-bit adjustment is also controlled by an off-chip current source. The threshold variation is $\sim 350 \mathrm{e}^{-} \mathrm{rms}$ before tuning and after tuning this falls to $\sim 80 \mathrm{e}^{-}$rms. The minimum applicable threshold is $\sim 2000 \mathrm{e}^{-}$. A 15-bit pseudo-random counter or Linear Feedback Shift Register LFSR [7] is used in a similar manner to the MPEC chip [8]. This counter is incremented each time the discriminator exceeds threshold and provided the shutter is open. When the shutter is closed the recorded 15 bits are shifted serially to the end of the column for readout. A photo of the die (Fig. 2) shows that, due to a large guard ring around the chip is was not possible to tile an area larger than one chip without significant loss of sensitive surface between chips. In spite of its limitations this chip was the first large scale photon counting chip and, as such, was used in multiple applications. One of the most iconic images is of a tungsten wire twisted to form the first letter of the word Medipix and which was placed on the surface of a Si detector and exposed to beta particles from a ${ }^{90} \mathrm{Sr}$ source (Fig. 3). Many more serious images were taken and the chip permitted studies of the X-ray imaging properties of materials such as GaAs [9-12]. The same device also started to yield hints of potential for dose reduction in mammography when combined with a GaAs sensor [13]. It also enables the direct comparison of the imaging performance of different sensor materials [14].

\subsection{Medipix2}

The Medipix2 Collaboration was formed in 1999 with the aim of developing a larger chip with significantly smaller pixel pitch $(\sim 50 \mu \mathrm{m})$ using $250 \mathrm{~nm}$ CMOS and to explore spectroscopic X-ray imaging for the first time. Due to a compromise between circuity complexity and the required area the Medipix 2 chip had a pixel pitch of $55 \mu \mathrm{m}$ and is composed of $256 \times 256$ pixels [15]. The schematic diagram of the pixel is shown in Fig. 4. In contrast to its earlier sibling, Medipix2 has 2 thresholds permitting window discrimination. Many details of the design can be found in [16]. The pixel front-end architecture chosen is based on the Krummenacher scheme [17]. This scheme has many benefits and has proven to be extremely flexible and robust. In particular, the circuit can compensate for detector leakage currents of either polarity of sensor diode. An externally-defined voltage (provided by an on-chip DAC) fixes the dc potential of the preamp output node and thus permits the appropriate choice of operating point dependent on the charge collection species. (The output is fixed at a high value for hole collection and a low value for electron collection providing the maximum dynamic range possible.) The main drawback of the Krummenacher scheme is that the noise generated by the bias current of the leakage current compensation network is essentially added in parallel to any shot noise coming from the sensor. The measured gain is $\sim 10.5 \mathrm{mV} / \mathrm{ke}^{-}$. The output of the preamp is then fed to the input stages of 2 separate discriminators. The discriminator scheme chosen was based on a differential pair producing a current proportional to the difference between the preamp output voltage and the threshold voltage, $\mathrm{V}_{\mathrm{TH}}$, which is produced by a 10-bit on-chip DAC. On-pixel threshold tuning is achieved by adding (using a 3-bit DAC) more or less current to the OTA output prior to a zero-crossing discriminator circuit which switches output polarity as a function of the direction of the 


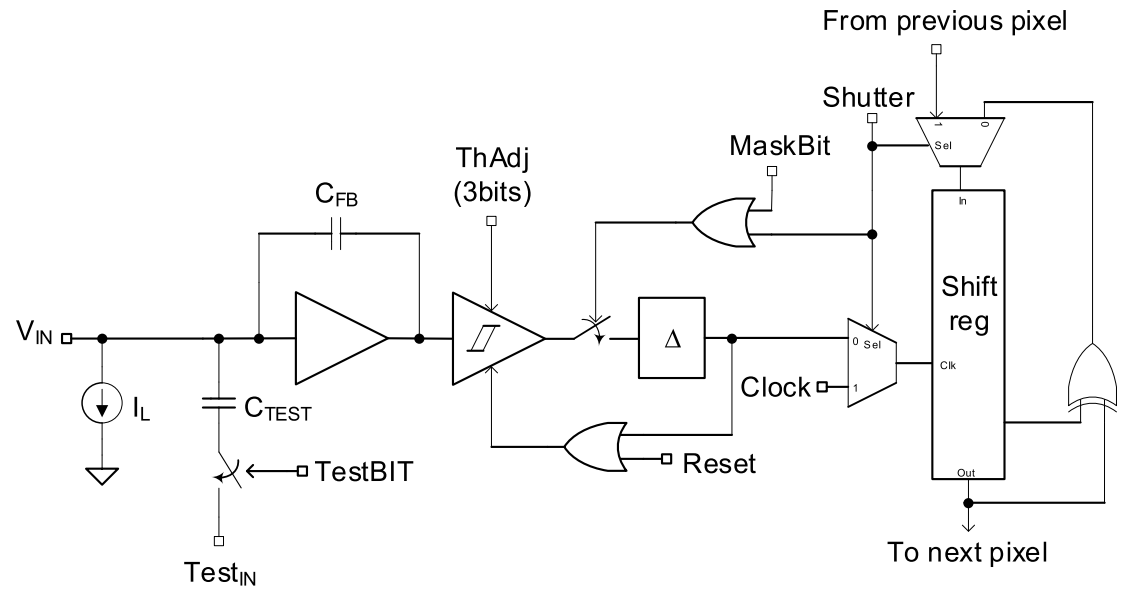

Fig. 1. Block diagram schematic of the Medipix pixel cell.

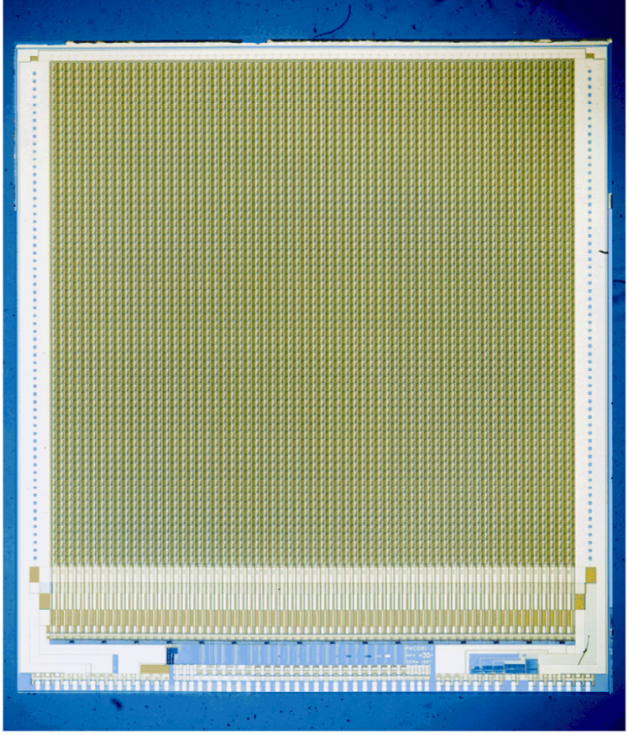

Fig. 2. Die photo of the Medipix chip.

incoming current. The output pulse of the high and low discriminators are then fed to a double discriminator logic. Provided the measured charge falls between the high and low discriminator values the circuit generates a pulse used to increment a 13-bit LFSR. A number of versions of the chip were fabricated each time improving on some specific feature. The final version of the chip is called MPIX2MXR2.0 and includes an on-chip bandgap reference circuit in the periphery, DACs with very low temperature dependence and a 14-bit on-pixel LFSR which stops on overflow at a counter value of 11810 . This chip was used extensively in many applications, mainly bump-bonded to a silicon pixel detector [18]. Flip-chip bump bonding of the Medipix2 chip with high density compound semiconductor detectors paved the way for hard X-ray and gamma-ray imaging [19]. One of the primary aims for Medipix2 was to investigate the potential and limits of spectroscopic $\mathrm{X}$-ray imaging at small detector pitches. Earlier simulations had indicated that charge sharing, as a consequence of charge diffusion during collection and/or fluorescence during charge deposition, would impose severe constraints [20] and indeed these limitations were confirmed by measurements using Medipix2 [21,22].

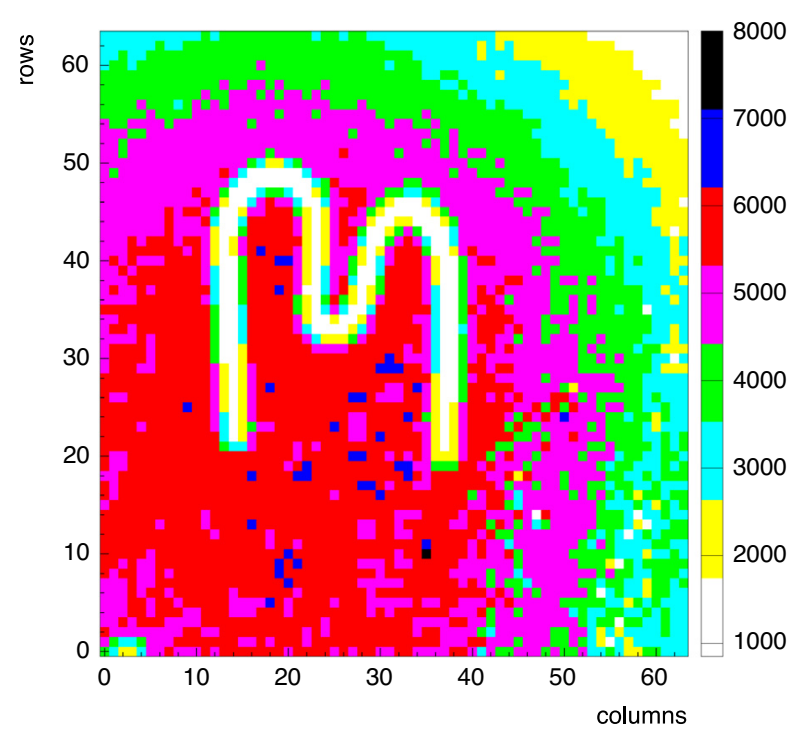

Fig. 3. Image of a tungsten wire shaped like an M (for Medipix) taken using the Medipix chip bump bonded to a $300 \mu \mathrm{m}$ thick silicon sensor and exposed to a ${ }^{90} \mathrm{Sr}$ source.

\subsection{Medipix3}

The Medipix3 Collaboration was formed in 2005. At that point we started to work on the implementation of ideas which would permit spectroscopic X-ray imaging even at relatively small pixel pitches [23]. In the case where soft X-rays ( $\sim 10 \mathrm{keV})$ are to be detected and a silicon detector is used charge sharing between neighbouring pixels due to charge diffusion is the primary mechanism leading to the misallocation of hits to lower energy bins. For a uniform exposure to a monochromatic source this results in a 'tail' in the single pixel spectrum towards lower energies. In the case of higher energy X-rays (25-100 keV) a higher-Z material than $\mathrm{Si}$ must be used to allow improved detection efficiency. In this case, the generation of fluorescence photons within the sensor material -with themselves a mean free path similar to a pixel pitch - is common and also leads to augmented charge sharing. The architecture we chose had to take both effects - charge sharing due to diffusion and charge sharing due to fluorescence in the charge deposition process into consideration.

A first small prototype chip was designed [24] and implemented in a $130 \mathrm{~nm}$ CMOS process. The chip had a matrix of $8 \times 8$ pixels on a pitch of $55 \mu \mathrm{m}$. Many details of that circuit can be found in [25]. The schematic diagram is shown in Fig. 5. Each pixel contains a charge sensitive 


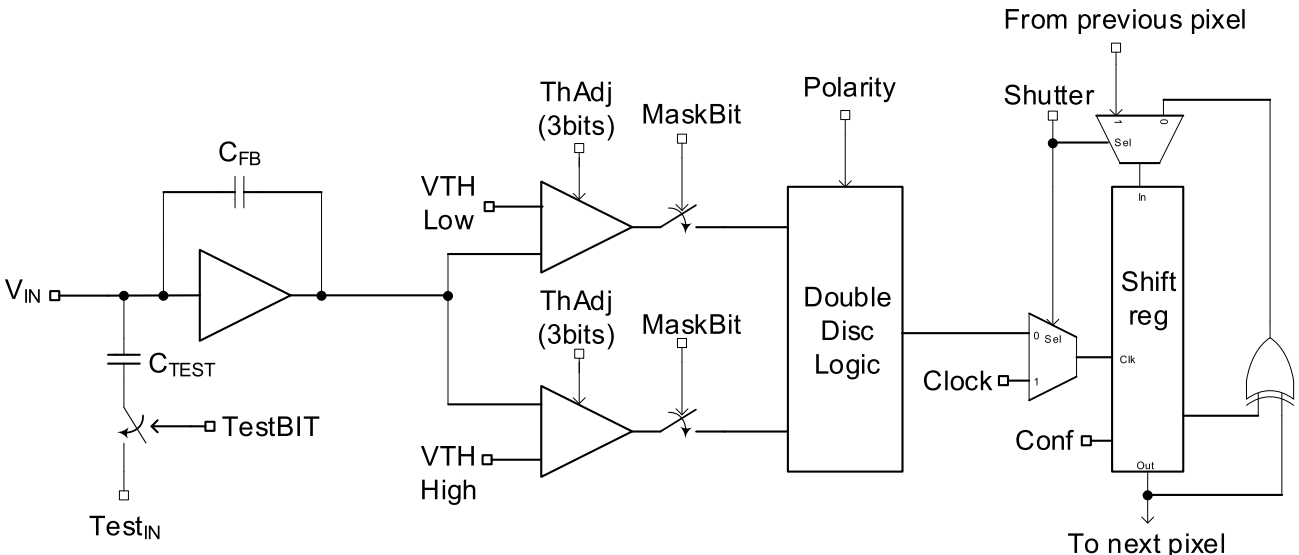

Fig. 4. Block diagram schematic of the Medipix2 pixel cell.

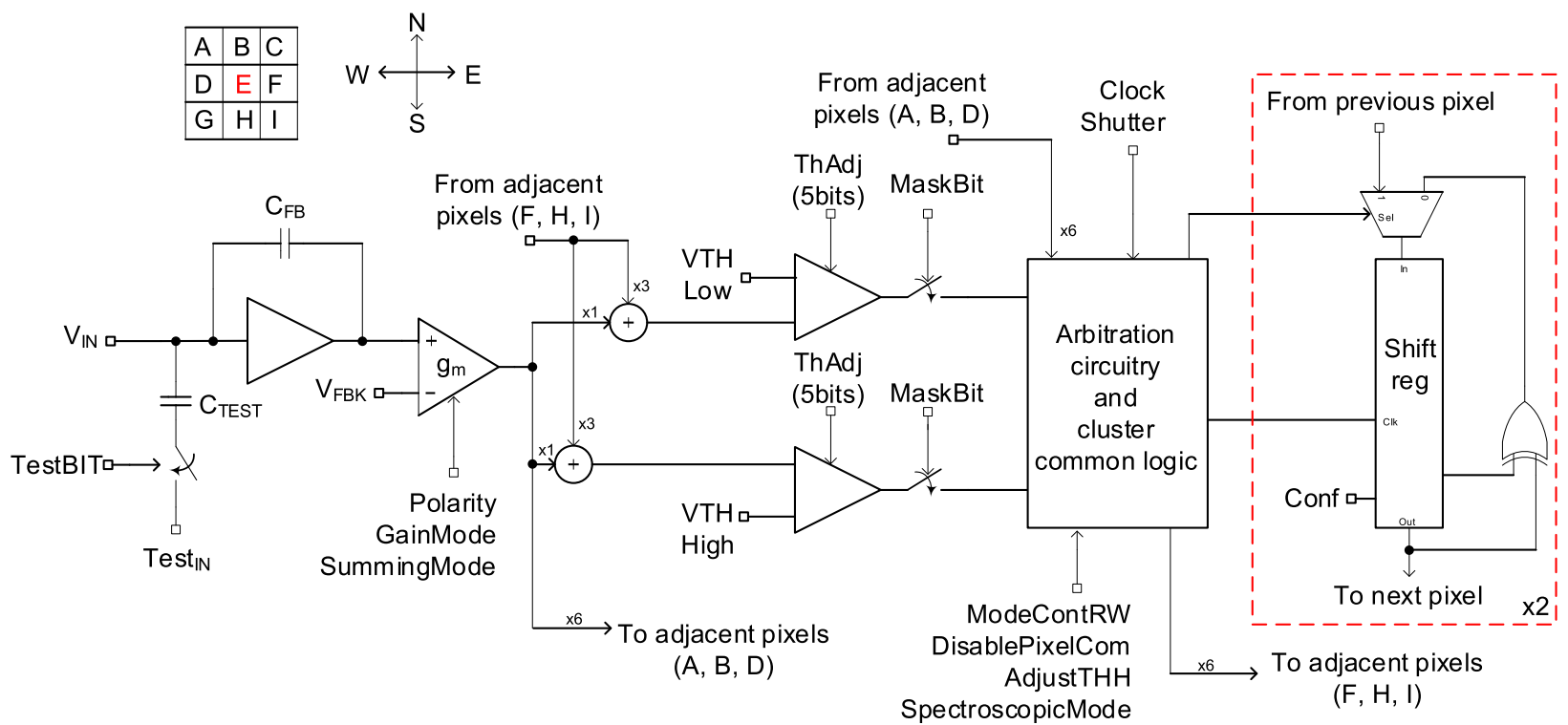

Fig. 5. Block diagram schematic of the Medipix3 prototype pixel cell.

amplifier using a front-end of the Krummenacher type, permitting both electron and hole collection. The output of the preamp is connected to an AC-coupled Gm-C shaping circuit whose time constant is $~ 100 \mathrm{~ns}$. Pole-zero cancellation is also implemented. The output of the shaping stage is a current proportional to the amplitude of the charge collected in a single pixel. Most importantly, this current is then copied multiple times and fed to different charge summing circuits. The summing nodes on a given pixel are connected to the shaping circuits of the pixel itself as well as to copies of the currents of its E, SE and S neighbours in the pixel matrix corresponding to the sum of the pixel's SE corner. The output of each summing circuit is fed to a discriminator, one for the high threshold and one for the low threshold. The discriminator outputs are then fed to the Cluster Common Control Logic and Arbitration Circuit. This circuit compares the discriminator outputs from those of the summing circuits in pixels $\mathrm{A}, \mathrm{B}$ and $\mathrm{D}$ (corresponding to the decisions related to the sums of pixels at the NE, NW and SW corners) identifying the corner with the greatest hit. Provided the local arbitrators have allocated the hit to pixel E the charge sum associated with the corner (NW, NW SW or SE) with the highest total charge is then used to increment the low counter if the sum is greater than the low threshold, and the high counter if it also exceeds the high threshold. As there are 215 bit LFSRs per pixel it is possible to programme the pixel to read out only one discriminator using only one counter at a time (the other discriminator being ignored). In that case 'continuous read/write' is available meaning that one counter is being read out while the other is being incremented. Another important feature of the prototype chip is that it can be programmed in spectroscopic mode. In this case only one front-end in 4 is connected to a sensor pixel with a pitch of $110 \mu \mathrm{m}$. Each super-pixel has 8 counters and 8 thresholds. Charge summing can be activated over 4 super-pixels (thus collecting charge over a total area of $220 \mu \mathrm{m} \times 220 \mu \mathrm{m}$ ) and the number of thresholds can be reduced from 8 to 4 permitting continuous read/write.

The success of the prototype chip led to the development of the first full Medipix3 readout chip [26] and this was the first large scale chip in $130 \mathrm{~nm}$ CMOS in the High Energy Physics community. This chip has a matrix of $256 \times 256$ pixels on a pitch of $55 \mu \mathrm{m}$ and, like the prototype chip, it can also be configured to a matrix of $128 \times 128$ pixels on a pitch of $110 \mu \mathrm{m}$. The pixel front-end is almost identical to that of the prototype chip. On the other hand, the counters were redesigned to allow more flexibility depending on the application. Each $55 \mu \mathrm{m}$ pixel can be programmed to have $2 \times 1$-bit counters, $2 \times 4$-bit counters, $2 \times$ 12-bit counters or a single 24-bit counter all of binary ripple type [27]. The results of measurements were all consistent with expectations with the notable exception of the pixel-to-pixel threshold variation. Even after tuning this was around a factor 3 higher than expected. This led to some pixel corners 'grabbing' hits which belonged to neighbouring corners [28]. Another undesired feature of this chip was the tendency of single pixels to spontaneously change threshold for some time (with 


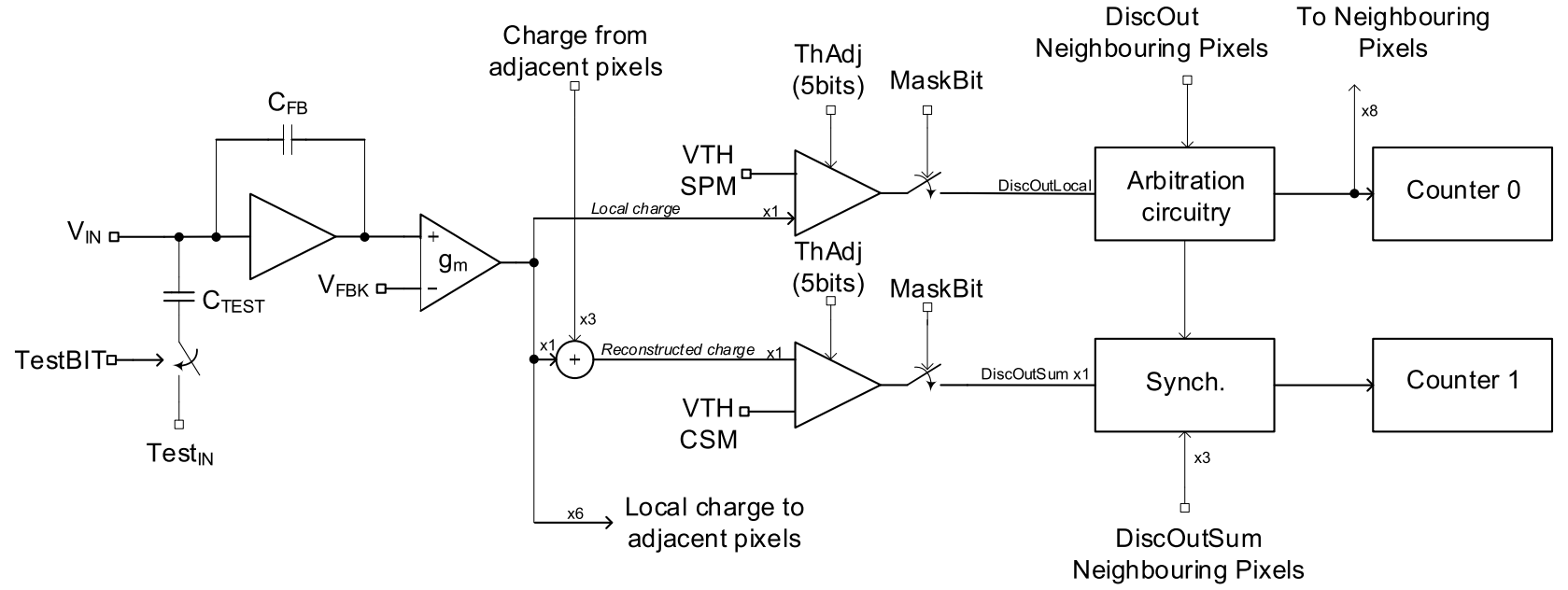

Fig. 6. Block diagram schematic of the Medipix3RX pixel cell.

a time constant varying from $\mu$ secs to hours) in a manner consistent with Random Telegraph Noise. A further observation was that the 8 threshold voltage DAC's (which were schematically identical) tended to have different gain slopes within a chip and from chip to chip. After much investigation, the origin of all of these problems was found to be related to degradation of the integrity of the thin gate oxide during fabrication resulting from an absence of tie-down diodes at Metal-1 on the analog matched pairs. In fact, the layout was fully consistent with the antenna design rules provided by the foundry but did not follow a recommendation related to tie downs for matched pairs. This effect became visible due to the downscaling of the gate oxide thickness from $\sim 5 \mathrm{~nm}$ (for Medipix2 and Timepix) to $\sim 2 \mathrm{~nm}$ and also the large number of pixels in the chip.

The Medipix3RX chip [29] was a major redesign of the first version. As well as resolving the mismatch problem it also incorporated a number of architectural enhancements. Among those was the decision to allocate a hit no longer to the pixel corner with the highest charge sum, but rather to the individual pixel in the neighbourhood with the largest local charge deposit [30]. For completeness, the schematic of the improved pixel cell is shown in Fig. 6 . This has the advantage that the decision is less prone to the impact of noise (the charge sum adds the noise of 4 pixels in quadrature) and also more precise allocation in the case of hits involving only one pixel. The disadvantage is that there may be hits missed in the case where charge is shared among many pixels and none is hit above threshold. In practice, however, the reduction in noise permitted operation at a lower minimum threshold and, in general, much improved results. It should be noted that a further limitation was that there are only 4 charge summed thresholds available in spectroscopic mode in both sequential read/write and continuous read/write modes. Fig. 7 demonstrates a measurement using a line pair mask and the Medipix3RX chip operating in both single pixel mode (left) and charge summing mode (right). There is no distinguishable difference in the line pair mask images indicating that charge summing has no impact to the spatial resolution. Fig. 8 shows the results of a threshold scan of the Medipix3RX connected to a CdTe sensor with a pitch of $110 \mu \mathrm{m}$ and $2 \mathrm{~mm}$ thickness when exposed to an ${ }^{241} \mathrm{Am}$ source [31]. Comparing the blue line (taken using Single Pixel mode) with the red line (taken using charge summing mode) one can perceive the power of the charge summing architecture in overcoming the dispersive impact of the charge deposition and collection mechanisms. Moreover, we believe that the small pixel effect [32] permits us to be sensitive only to one type of the drifting carriers (in this case electrons) and therefore diminishes the impact of charge trapping of the other species (the holes in this case) thereby improving energy resolution. An important innovation in the design approach for Medipix3RX was the use of a standard cell library within the pixel cell for the first time. This has the benefit of permitting precise simulations of the full readout architecture with a corresponding reduction of risk of introducing errors during the chip assembly process. Because of the circuit complexity permitted by the $130 \mathrm{~nm}$ CMOS process the standard cell design approach is now used systematically in new pixel design projects.

\section{Timepix chips}

In 2005, the Medipix design team was approached by members of the EUDet Collaboration who were interested in adapting the Medipix2 chip for gas detector readout. This followed on from earlier experiments showing that, when combined with a gas gain grid, the Medipix 2 chip was able to record the position of individual electrons produced by an ionising particle in a gas volume [33]. This request led to the development of the Timepix chip and ultimately to a new branch in the efforts of our Collaborations. These chips aim to extract the maximum amount of information possible concerning charged particle interactions in the associated sensor.

\subsection{Timepix}

The above-mentioned measurements with Medipix2 produced a 2D projection of a 3-D event in the gas volume. As an ionising particle traverses a gas volume individual photo-electrons are created and these drift under the influence of an electric field towards a gas gain grid. At the grid, the charge, if transmitted, is subjected to such a high electric field that charge multiplications takes place. The charge cloud at the output is then drifted by an electric field onto the pads of the readout chip where it is detected in a way similar to a charge cloud drifting in a semiconductor sensor. The Medipix2 chip simply counts hits above threshold while the shutter is open. In the example shown in [33] the image is composed of a matrix on ' 1 's and ' 0 's representing the projection of the 3-D track information onto the 2-D pixel matrix. The request from the EUDet consortium was to modify the Medipix2 pixel cell such that, instead of counting particles, the cell should count clock ticks from the moment the particle is detected until the moment the shutter is closed thus providing the 3-D track information. During discussions with the design team we realised that, if a clock is distributed to each pixel, then we could offer the option of measuring Time-over-Threshold instead allowing to measure the total collected charge. The schematic of the Timepix pixel is shown in Fig. 9 [34]. The pixel front-end is identical to the Medipix2MXR chip but there is only a single threshold. The on-pixel threshold adjustment DAC has 4 bits providing a minimum operating threshold of $\sim 600 \mathrm{e}^{-}$. The pixels can be programmed individually to either (a) count hits (b) count clock ticks from when the discriminator is fired until the shutter closes providing Time of Arrival information 

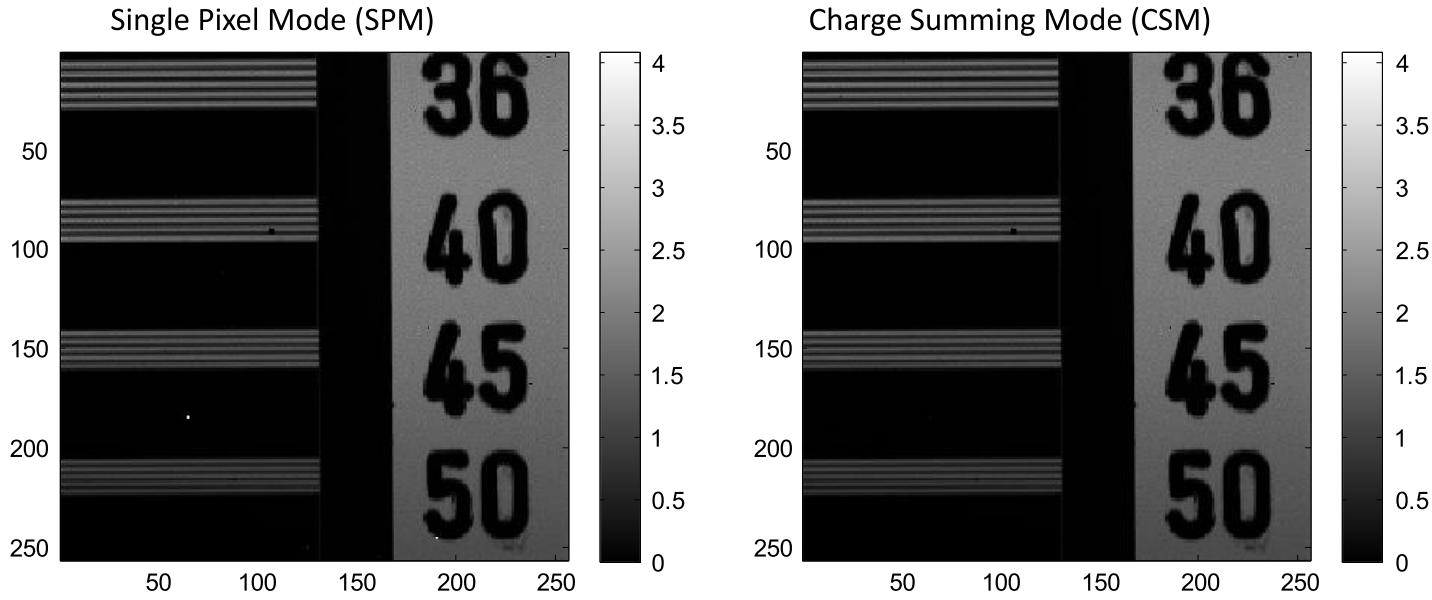

Fig. 7. Images of a line pair mask taken using an X-ray tube and the Medipix3RX chip in single pixel mode (left) and in charge summing mode (right).

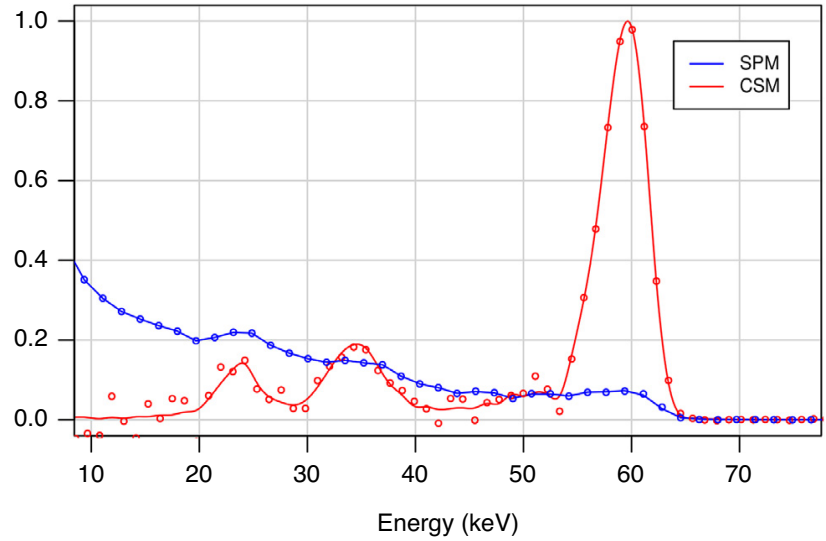

Fig. 8. Spectra reconstructed from a threshold scan based on raw data from all pixels using the Medipix3RX chip in single pixel mode (blue) and in charge summing mode (red) [31]. In CSM the photo-peak has $\sim 4 \mathrm{keV}$ FWHM. (For interpretation of the references to colour in this figure legend, the reader is referred to the web version of this article.)

or (c) count clock ticks while the threshold is exceeded providing Time-over-Threshold information. A single 14-bit LSFR which stops on overflow at 11810 is provided and the frame-based readout protocol is identical to Medipix2. Thus, Timepix could be read out with only slight modifications to the Medipix2 readout systems. The versatile nature of the chip has led to its application in many applications as outlined later.

\subsection{Timepix3}

Although the Timepix chip is very widely used it has a number of important limitations. When measuring Time of Arrival (ToA) the counter starts when the discriminator passes threshold and stops when the shutter closes. This limits the shutter opening time to the period of the clock multiplied by the counter depth (11810). In Time-overThreshold (ToT) mode a second particle strike on the same pixel while the shutter is open will result in the ToT values of both hits being added and hence an error in the measurement. Moreover, each pixel can record either ToT, ToA or the number of hits. Also, during the framebased readout the pixel matrix is insensitive. These limitations led to the specifications for the Timepix 3 chip.

Unlike all of the other chips in the Medipix/Timepix family, Timepix3 has a data-driven architecture [35]. When a pixel is hit it immediately initiates the sending of its data off-chip. A schematic of the Timepix3 pixel is shown in Fig. 10 [36]. The front-end amplifier follows the usual Krummenacher scheme and there is a single discriminator with 4-bits of threshold adjustment [37]. The tuned threshold variation is $\sim 30 \mathrm{e}^{-}$rms providing a minimum threshold of $\sim 500 \mathrm{e}^{-}$. The output pulse of the discriminator is used to start the 4-bit fine time stamp counter and to latch the 14-bit time stamp provided by an on-chip $40 \mathrm{MHz}$ Gray-encoded ramp counter. There is also a 10-bit pseudorandom counter running at $40 \mathrm{MHz}$ which records the ToT. The back end of the digital part of the pixel groups $2 \times 4$ pixels into super-pixels. Each of these super-pixels has a single Voltage Controlled Oscillator (VCO) which is shared between the 8 pixels [38]. The VCO is started by the $\mathrm{OR}$ of the rising edge of the discriminator outputs and oscillates at a frequency of $640 \mathrm{MHz}$ until the next rising edge of the $40 \mathrm{MHz}$ clock. The fine timestamp on a given pixel is generated by counting the number of VCO clock ticks from the moment that the discriminator passes threshold until the VCO stops. Data from a given pixel is passed to the super-pixel using round robin arbitration. The deadtime associated with clearing the digital information from the pixel is $475 \mathrm{~ns}$. Hit information is passed from the super-pixel to the bottom of the column by a token ring scheme providing access to one super-pixel at a time to a 10-bit parallel readout bus. The information sent comprises the super-pixel address ( 6 bits) the hit pixel address within the super-pixel (3 bits), course ToA (14-bits), Fine ToA ( 4 bits) and ToT (10 bits). In total, each hit generates a data word of 44 bits plus a 4-bit header which are sent off chip.

\section{Other ASIC developments related to the Medipix efforts}

\subsection{Dosepix}

The background to the Dosepix development lies with ideas generated within the context of the Medipix2 Collaboration by people interested in making a chip suitable for X-ray dosimetry [39]. The basic concept was to design a chip composed of a matrix of pixels each containing a multi-channel analyser circuit. The Dosepix chip contains a matrix of $16 \times 16$ pixels at a pitch of $220 \mu \mathrm{m}$ [40,41]. Many details of the circuit can be found in [42]. The pixel has three main operating modes: particle counting, energy integrating and dosimetry mode. In particle counting mode (intended mostly for calibration purposes) the pixel simply counts the number of hits above a predefined analog threshold using an 8-bit counter (something akin to the operation of Medipix). In energy integration mode a 24-bit counter records the total ToT for a given pixel. The most useful mode however is the dosimetry mode. In this case, a 12-bit ToT counter records hits one-by-one. When the discriminator goes low after a given hit the contents of the counter are latched and then compared with 16 programmable on-pixel thresholds. When the comparison shows that a hit lies within a given threshold window a 16-bit energy window counter is incremented. In this way, the spectrum of hits on a given pixel is recorded with 16 energy bins. 


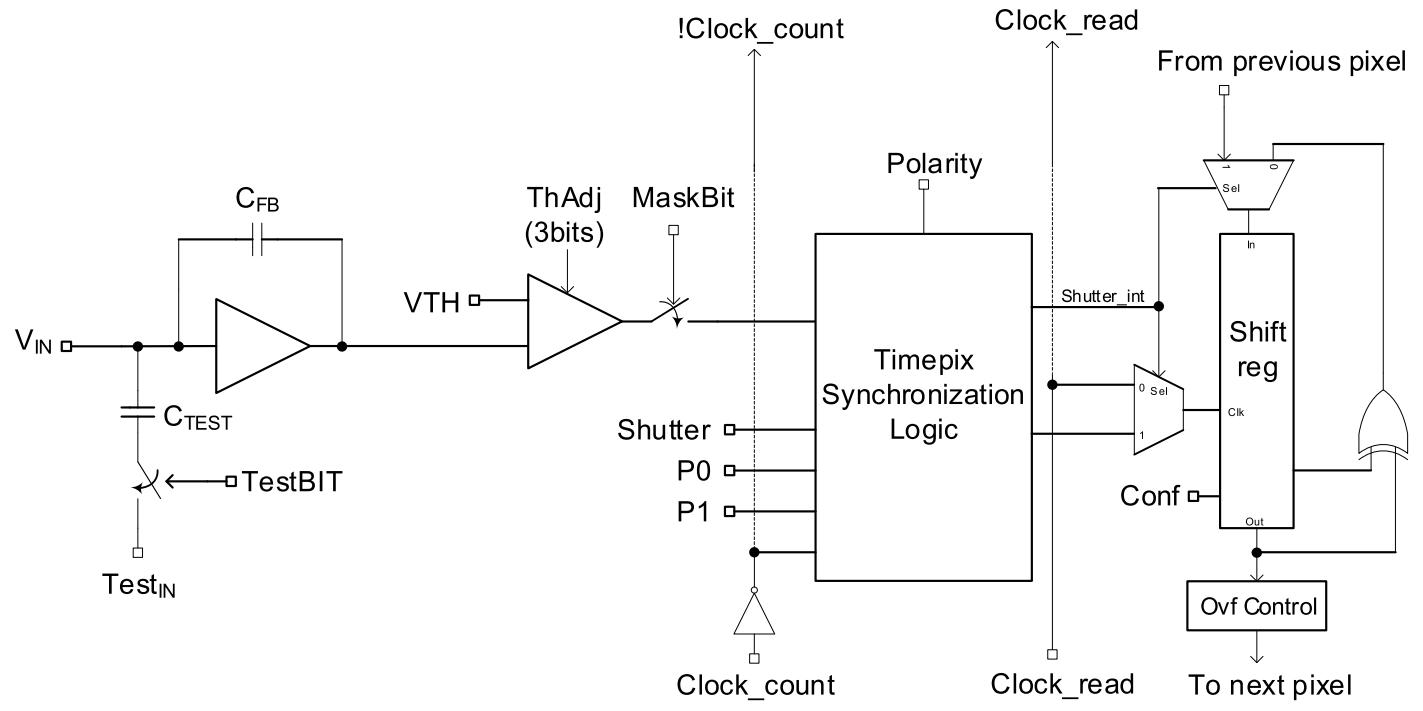

Fig. 9. Block diagram schematic of the Timepix pixel cell.

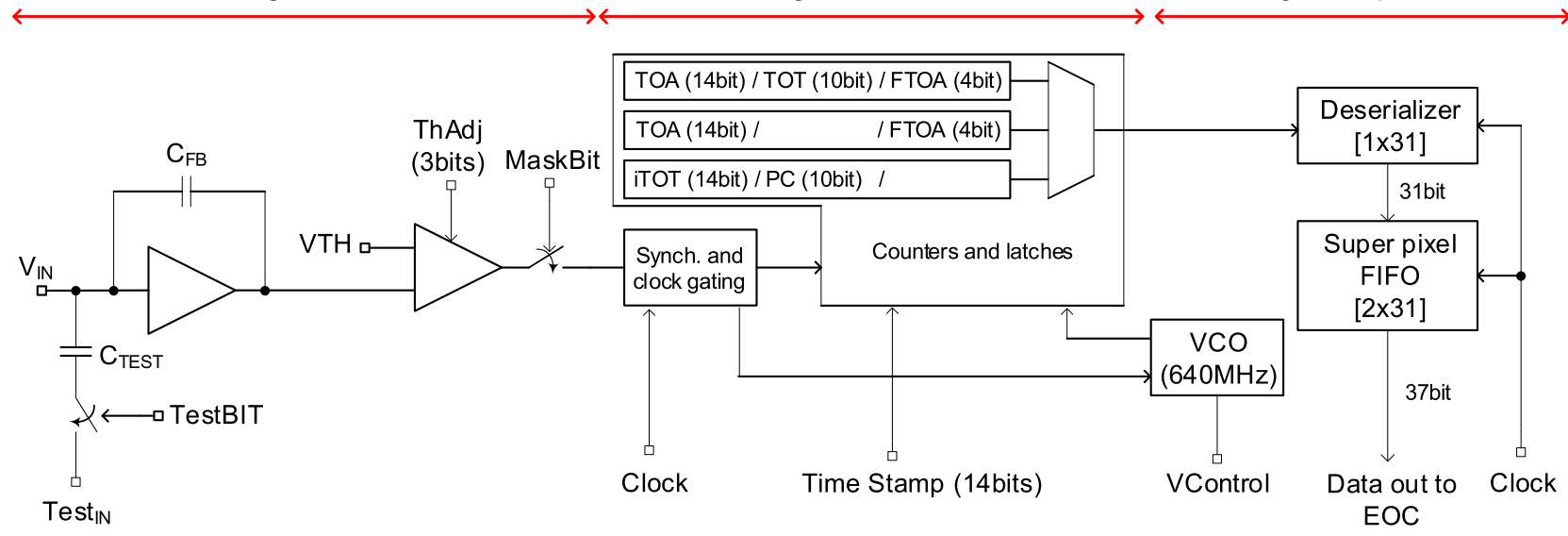

Fig. 10. Block diagram schematic of the Timepix 3 pixel cell.

Note also that the energy thresholds can be programmed differently from pixel to pixel which means that the chip can have a total of 16 $\times 16$ energy thresholds. Readout is performed on a column by column basis. While one column is being read out all 15 others are continuously sensitive.

\subsection{VELOpix}

The Timepix ASIC was incorporated into a 6-plane charged particle beam telescope [43] and became a workhorse for testing various new detector structures for charged particle tracking. With $55 \mu \mathrm{m}$ square pixels and then tilting the assemblies with an angle of $\sim 9^{\circ}$ it was possible to obtain a pointing resolution of down to $2.4 \mu \mathrm{m}$ by making full use of the ToT information in charge shared events. As well as being the ASIC used for the telescope, the Timepix chip was also used as the readout for various new kinds of detector structure (3-D detectors, ultrathin detectors, edgeless detectors). As indicated above, however, one of the primary limitations of Timepix is its frame-based readout. In the telescope the shutter opening time had to be tuned such that no pixel was hit twice during a data taking run which in practice limited the number of hits in one frame to about 100 tracks. After that time, the telescope is essentially dead while the entire contents of the matrix are read out.
Timepix 3 resolved many of these issues and it has also been successfully incorporated into a charged particle beam telescope running with a rate capable of reading out every particle track provided by the CERN SPS $\mathrm{H}-6$ beamline (LHCb publication in preparation) at full beam intensity.

Timepix 3 demonstrated that noise-free trigger-less readout could be achieved with pixels on a $55 \mu \mathrm{m}$ pitch and with a threshold of $\sim 500 \mathrm{e}^{-}$. The Timepix3 chip provides a 48-bit word for each pixel hit and this, combined with the output chip bandwidth of $5.2 \mathrm{~Gb} / \mathrm{s}$ ultimately limits the flux of particles which can be processed correctly. Timepix3 can deal with $\sim 80$ Mhits/s. The VELO upgrade for the LHCb experiment [44] requires the chips nearest to the particle beam to detect up to 15 hits in every LHC bunch crossing. This fixes the upper limit for the bandwidth of the VELOpix chip and imposes some restrictions on the chip architecture. Also, the chip should work in a radiation environment and techniques such as Triple Redundancy are used to mitigate the effects of Single Event Upset. Such techniques also reduce the complexity which can be incorporated within a pixel. The VELOpix architecture is described in [45]. Each pixel has a preamplifier (optimised for electron collection) and discriminator with an associated 2-bit ToT counter. The pixel can be programmed such that hits below a given ToT value are ignored to help eliminate low energy hits near 
to threshold and therefore subject to timewalk. Validated hits are timetagged to the $40 \mathrm{MHz}$ LHC clock by latching the contents of 9-bit Grayencoded Bunch Crossing Counter. The pixels are organised into superpixels of $2 \times 4$ pixels. If at least one pixel is hit the binary map of the super-pixel along with the timestamp is latched and prepared for readout. The initial electrical results from the chip indicate that full functionality has been achieved. However, measurements with bumpbonded sensors and radiation tolerance are still under-way.

\subsection{CLICpix}

Looking towards accelerators beyond the LHC one option being studied is the CLIC linear electron-positron collider [46]. At CLIC, bunch trains of electrons and positrons will collide at $20 \mathrm{~ms}$ intervals. Each bunch train will comprise around 300 bunches spaced by 0.5 ns. At most one hard interaction is expected per bunch train. However, at the highest CLIC energy of $3 \mathrm{TeV}$ many beam-induced background particles are expected. To maintain low occupancies, even in the hottest region of the vertex detector, pixels of $25 \times 25 \mu \mathrm{m}^{2}$ are foreseen. Background suppression furthermore calls for $10 \mathrm{~ns}$ time stamping capabilities. The physics requirements aim for $3 \mu \mathrm{m}$ spatial resolution and a very low material budget of $0.2 \%$ of a radiation length per layer including services and supports. The CLICpix chip has been designed in this context, using $65 \mathrm{~nm}$ CMOS and a pixel pitch of $25 \mu \mathrm{m}$ [47]. This was the first pixel chip to be designed in $65 \mathrm{~nm}$ CMOS and paves the way for future developments both for CLIC and for the Medipix4 collaboration.

\section{Applications}

One of the outstanding features of the Medipix and Timepix family of ASICs are the multiple applications to which they have been dedicated by the (more than 30) different teams participating in the Medipix collaborations. Some of these are commercial but many are of a scientific nature. For applications concerning Medipix2 prior to 2009 the reader is invited to consult this reference [18]. It would be unreasonable to try to provide a comprehensive list here but we will highlight some of major applications in what follows.

\subsection{Educational purposes}

The availability of a compact readout systems [48-50] and user friendly software [51] for the Medipix and Timepix chips greatly facilitates access to these devices. Following a visit by a group of high school students from the Simon Langton School in Canterbury, England to the Medipix design team at CERN in 2007 there was great enthusiasm for the potential to apply such devices both in the classroom and for other school-based projects. The students started to use the Medipix2 and then the Timepix devices in the classroom. They also proposed the construction of a satellite system based on Timepix and this was ultimately flown by Surrey Satellite Technology on their TechDemoSat1 mission [52]. In the meantime, many experiments have been carried out by high school students using these devices and anecdotal data suggests that such activities strongly influence the subsequent choice of students towards the STEM subjects at university. In March 2016, the Institute for Research in Schools (IRIS) was officially launched at the Science Museum in London. Many of the initial projects proposed by IRIS are based on the use of the Timepix chip. At the time of writing over 300 schools have subscribed to the IRIS programme whose aim is to stimulate the interest of high school students in studying science and engineering by introducing them early to the real research environment. The Medipix2 and Medipix3 Collaboration will continue to support this important initiative and any other similar activities related to science education.

\subsection{Space dosimetry and space weather}

In parallel with the school-initiated satellite project a number of activities have gone on in the US and in Europe on space-based dosimetry and space weather.

The US-based activity has been spear-headed by the University of Houston group with strong technical support from the Institute of Experimental and Applied Physics of the Czech Technical University and more recently from ADAVACAM sro (a spin-off company from CTU). They have been responsible for the installation and maintenance of 7 Timepix-based detectors on the International Space Station (ISS). An example of a map of radiation dose measured by the Timepix devices on the ISS is shown in Fig. 11. The successful (and reliable) operation of these devices $[53,54]$ persuaded NASA to incorporate 2 Timepixes on the Orion test vehicle launch in December 2014. Those devices also worked well and NASA has base-lined the approach for future missions of the Orion manned spacecraft.

The ESA-sponsored project SATRAM (Space Application of TIMEPIXbased Radiation Monitor) uses the Timepix detector to continuously monitor space weather $[55,56]$. It is incorporated in the ProbaV earth monitoring satellite in a sun-synchronous orbit at an altitude of $820 \mathrm{~km}$ and monitors the direction and stopping power of incoming charged particles.

\subsection{Synchrotron imaging}

Photon counting systems have been widely adopted at synchrotron light sources over the last 15 years. Among the first systems to be used regularly at a beamline is the PRIAM readout system which uses the Medipix2 chip for photon counting [57] and which acted as a precursor to the MAXIPIX system [58] which is capable of reading out up to 5 Medipix2 or Timepix chips. In both cases the Medipix2 (or Timepix) chip is read out using the 32-bit parallel bus at a clock frequency of $100 \mathrm{MHz}$. This reduced the readout (or dead) time to less than 300us and therefore provided $\mathrm{kHz}$ framerates. The MAXIPIX system has most recently been incorporated in a novel facility at ESRF providing X-ray Raman scattering spectrometry [59].

At DESY recent work has focussed on the development of the large area LAMBDA system which is based on Medipix3RX [60]. That system is composed of tile-able Si detector modules with $1536 \times 512$ pixels and runs at 2000 frames per second. Each detector module is connected to $6 \times 2$ Medipix3RX chips. The LAMBDA system makes full use of the deadtime free operation of the Medipix3RX chip. Individual tiles using $\mathrm{GaAs}$ or CdTe as a sensor material can also be provided where the photon energy to be used requires a more efficient detector material.

At the Diamond Light Source two systems have been developed. The Merlin system [61] is a compact, LabView based readout which is aimed at small detector areas (up to 4 chips). It was first designed for Medipix 3 and has since been adapted to Timepix3 [62]. The other major activity at Diamond is the development of the EXCALIBUR readout [63]. This Medipix3RX-based system is made up of 3 individual modules each comprising a silicon ladder connected to 16 readout chips. The entire system of $3 \mathrm{M}$ pixels can be readout out at 100 frames per second in continuous mode or 1000 frames per second in burst mode.

\subsection{Studies of sensor materials}

Silicon is the sensor material of choice for most High Energy Physics experiments. Historically sensor thicknesses of $300 \mu \mathrm{m}$ using wide guard rings were used. However, recent developments in Silicon processing have permitted either very thin $(50 \mu \mathrm{m})$ or very thick $(>500 \mu \mathrm{m})$ sensors to be used. Moreover, sensors with little or no insensitive edges are of particular interest in tracking detectors or where large areas have to be tiled seamlessly. The availability and flexibility of Medipix2 and Timepix has permitted a large number of studies of the behaviour of thin and thick sensors both with and without active edges [64]. 


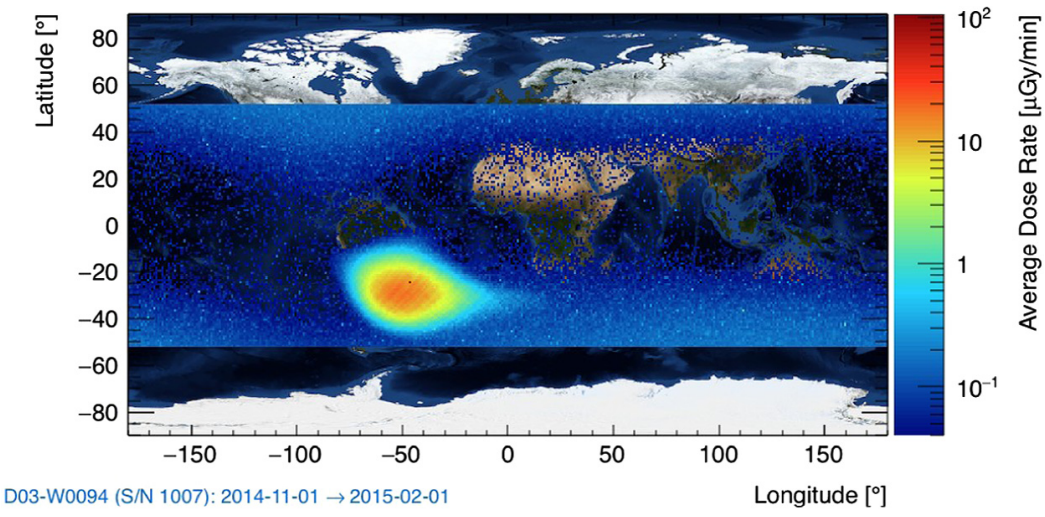

Fig. 11. Image reconstructed from data from one of the Timepix assemblies on board of the International Space Station (courtesy L. Pinsky, University of Houston).

High-Z material are of particular interest in X-ray imaging applications where the mean photon energy exceeds $\sim 15 \mathrm{keV}$. In this case GaAs, CdTe and CdZnTe become very interesting candidate materials. In a similar way to the Si sensor studies, the Timepix chip has acted as the host to many different candidate sensor materials providing invaluable insight into the distribution of defects and the charge collection mechanisms [65-67]. The Medipix3RX chip has also been used for the study of GaAs material [68] and for time dependent variations in CdTe material [69].

\subsection{Spectroscopic X-ray, gamma-ray and particle imaging}

As mentioned earlier the detection of X-ray photons one-by-one permits the development of systems capable of high resolution spectroscopic X-ray imaging. With the Timepix chip it is possible to measure the total charge deposited in a cluster of pixels and therefore correctly bin the detected photon according to the total charge deposited [70]. A specific application was counting or spectroscopic imaging of radiation quanta (electrons, positrons, gamma-rays, neutrons) with the Timepix chip [71-75]. More recently a large area Timepix-based detector (consisting of 100 chips) has been used for high contrast X-ray microradiography of histology samples [76]. When the Timepix chip is used in ToT or ToA mode the primary limitation comes from the need to avoid overlapping clusters and in practice this reduces the number of cluster per frame and hence the frame length. Moreover, as the Timepix chip is insensitive during readout this imposes strong restrictions on the applications. As already mentioned, the Timepix 3 chip overcomes many of these limitations by using a data-driven architecture. Timepix 3 is capable of treating up to 80 Mhits per second and therefore an incoming flux corresponding to $80 \mathrm{MHz}$ divided by the number of hits generated by each photon in the sensor.

However, in even higher rate applications the inter-pixel charge summing and allocation scheme first implemented in the Medipix3 chip must be used. The benefits of the charge summing and allocation scheme have been studied and proven experimentally [77]. There are many examples of its use in materials analysis [78] and medical imaging $[79,80]$. One particularly exciting medical application is spectral CT imaging using metal nanoparticles which are attached to biological tracers [81]. It may be that this could lead to functional imaging in radiology encroaching on a domain which has so far depended on nuclear medicine. Fig. 12 shows a CT scan of a mouse taken by the MARS team in New Zealand showing a good separation of Gold, Gadolinium and Iodine in clinically-acceptable concentrations.

\subsection{Electron microscopy}

Direct detectors, such as those available in the Medipix and Timepix families offer substantial benefits in terms of efficiency and point spread function when compared with conventional indirect detectors in

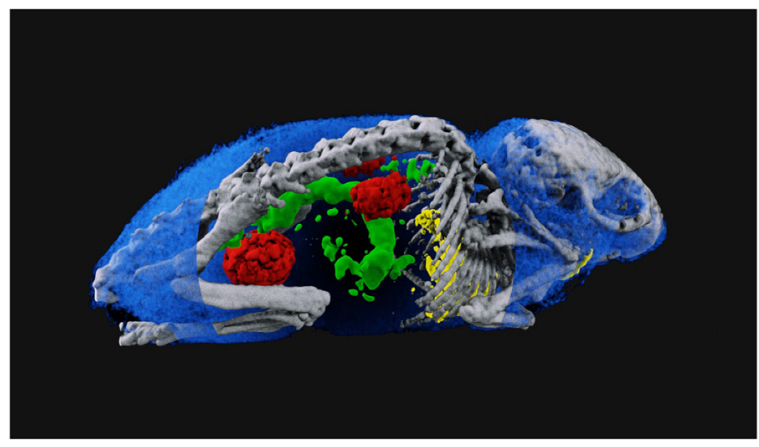

Fig. 12. Image of a mouse injected with biomarkers with doped with Gadolinium, Gold and Iodine metal nano-particles (courtesy of A. Butler, University of Canterbury, Christchurch, New Zealand).

electron microscopes. CCDs offer high pixel counts but typically suffer from relatively slow frame-based readout. Back illuminated and fully depleted Monolithic Active Pixel sensors provide more sensitivity but also tend to have limited frame rate and can be damaged by electrons with enough energy to reach the electronics unless special radiation tolerant design techniques are adopted. Hybrid pixel detectors tend to have larger pixels but with sharp point spread functions and faster readout speed. This was demonstrated already in 2003 with Medipix [82] and then later with Medipix2 [83]. Rather spectacular results were obtained using Medipix2 in Low energy Electron Microscopy in 2009 also [84] as illustrated by Fig. 13. However, with increased electron energy ( $>200 \mathrm{keV}$ ) the scattering of the electrons within the relatively thick sensor limits the point spread function [85]. Recent work at Glasgow using Medipix3RX has indicated that the charge summing and allocation scheme can provide a substantial improvement in point spread function [86]. It has also been postulated that using Timepix3 and reconstructing the track in 3-D it should be possible to identify in most cases the impact point of each electron on the sensor thus providing an almost perfect detector for electron microscopy. Preliminary laboratory studies by the authors using a ${ }^{90} \mathrm{Sr}$ source appear to confirm this.

\subsection{Axion search and other gas based detector systems}

The Timepix and Timepix 3 chips are being used to record the charge arising from an avalanche in a high electric field. Following the Micromegas principle, an amplification grid is mounted $50 \mu \mathrm{m}$ above the Timepix chip. The grid is perfectly aligned with the pixels by and supported by insulating pillars by means of wafer-based photolithography (InGrid, GridPix). The charge-sensitive amplifiers on the Timepix are efficiently protected from discharges by a thin $(2-8 \mu \mathrm{m})$ protection layer. GridPix detectors can be exploited for charged particle 

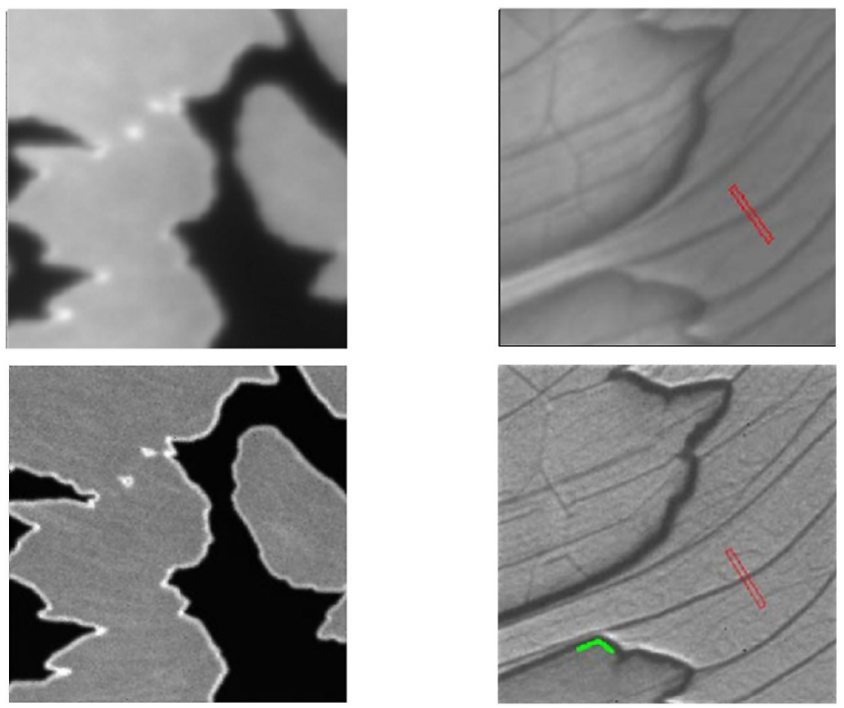

Fig. 13. Low Energy electron microscope images comparing the growth of graphene flakes. Upper images taken form the standard MCP + CCD imager, the lower images are taken with a Timepix assembly with silicon detector [84].

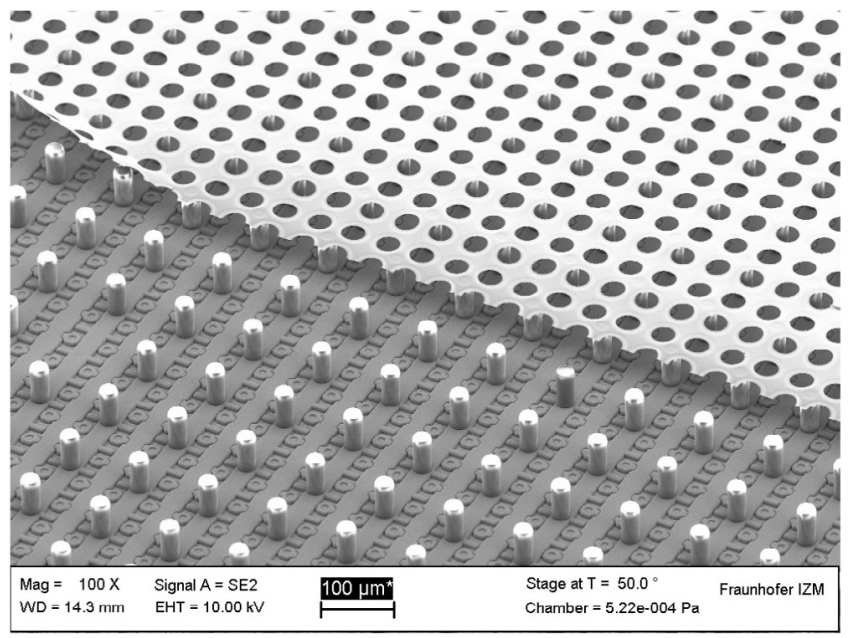

Fig. 14. Image in the InGrid integrated on top of the Timepix3 chip (courtesy of Y. Bilevych, Physikalisches Institut, University of Bonn, Germany).

tracking, e.g. in a large volume TPC planned for the ILD detector at the ILC. A large prototype, employing 160 Timepix-based InGrids has been constructed [87]. Although the resolution of the readout pixels is far in excess of that needed for the drifted (and thereby diffused) electrons in the gas volume the pixelated TPC detector provides clean readout at unprecedented spatial resolution for such a large gaseous detector. Moreover, the specific energy loss $(d E / d x)$ can be measured precisely from counting the number of individual electron clusters along the track. GridPix detectors have also recently been employed in the construction of a prototype for proton beam radiography [88].

A second application of Timepix-based InGrids is the detection of low energy X-rays and measurement of their energy by counting the number of electrons deposited. Very efficient background discrimination from topological analysis of the recorded events and very low energy threshold $(\sim 200 \mathrm{eV})$ make the detector suited for rare event searches. This has led to the implementation of such a detector at the CERN Axion Solar Telescope where it has been running reliably for many 100's of days. First InGrids based on Timepix3 have been produced (see Fig. 14) and will further extend the application of gaseous detectors.

\subsection{Micro Channel Plate imaging}

In a manner, much akin to the readout of gas gain grids it is possible to combine a naked readout chip with a Micro Channel Plate. An electron impinging on the front face of a MCP is multiplied as it traverses the pore of the device creating a charge cloud at the rear side. Provided an adequate electric field is applied between the rear of the MPC and the readout chip the charge cloud can be detected by the chip in much the same way as a charge cloud drifting in a semiconductor sensor. The MCP can be doped with a neutron absorber such as Boron for direct neutron imaging [89], providing unique contrast in neutron imaging experiments where other conventional methods fail due to sample opacity e.g. High-Z materials such as nuclear fuels, [90], inclusions of Pd within natural gold single crystals, [91] or have low contrast (e.g. hydrogenous substance such as oil imaged within shale samples). Alternatively, the MCP can be integrated into a tube with a photocathode for visible light imaging [89,92]. To make images of a medical or biological biopsy under laser ablation of molecules, the AMOLF team made use of Timepix devices in vacuum for Time-of-Flight mass spectrometry [93].

\subsection{Anti-matter research}

At the CERN Antiproton Decelerator at CERN the AEgIS experiment seeks to measure the gravitational pull on anti-hydrogen by studying the exact position at which it decays with a position sensitive detector. The Timepix3 detector has been evaluated for such a task as it is possible to use this combined with a thick silicon sensor as an active target in such a setup [94]. Anti-hydrogen atoms annihilate when they reach the detector surface and the resulting star-shaped events can be reconstructed off-line. Not only can the projections of the charged particles in $\mathrm{x}$ and $\mathrm{y}$ be recorded but a 3-D reconstruction can be carried out using the ToA information.

\subsection{Other applications}

The possibility to combine the readout chips described above with various sensor materials, gas detectors and microchannel plates has led to an explosion of uses not mentioned above. Among those are particle beam monitoring both at the CERN UA9 experiment and more recently for a Beam Gas Ionisation monitor [95] at the CERN PS. There is also an extensive network of Medipix2- and now Timepixbased radiation monitors active in the CERN ATLAS cavern $[96,97]$. The Timepix chip is used at the ISOLDE facility to study isotope deposition is crystalline structures using electron emission channelling. In the CERN Radioprotection group the effort has focussed on the development and use of the Timepix-based GEMPIX detectors to study hadron therapy beams and ${ }^{55} \mathrm{Fe}$ residues in radioactive waste. Other uses in medical applications include Timepix as a tracking detector for Hadron Therapy dose deposition monitoring $[98,99]$ and as an imaging system during LDR Brachytherapy seed implantation [100]. Timepix can also be used for nuclear physics experiments [101] and more recently to study the interaction of neutrons with a silicon sensor using Timepix3 [102]. Using ${ }^{10} \mathrm{~B}$ as a conversion layer and analysing the shape of incoming tracks it is possible to detect slow neutrons even in the presence of a high $\gamma$ and $\mathrm{e}^{-}$background [103]. A similar approach was used to construct a large scale neutron imaging system using ${ }^{6} \mathrm{LiF}$ as a convertor layer [104].

The probability to create an additional second vacancy in the K-shell of the manganese atom after electron capture decay of ${ }^{55} \mathrm{Fe}$ could be measured with unprecedented accuracy [105]. In this experiment, two Timepix detectors faced each other with the radioactive source in between them. The signal for double vacancy creation was the coincident detection of two x-ray fluorescences emitted during relaxation of the atomic shell, thus the triggering of two clusters of pixels with the same time-of-arrival. In addition to the measurement of the overall probability for this process, the pixelation of the sensor matrix was exploited to 
Table 1

A summary of the main features of the chips in the Medipix and Timepix family.

\begin{tabular}{|c|c|c|c|c|c|}
\hline & Medipix & Medipix2 & Timepix & Medipix3 & Timepix3 \\
\hline Pixel side $(\mu \mathrm{m})$ & 170 & 55 & 55 & $55 / 110$ & 55 \\
\hline Technology (nm) & 1000 & 250 & 250 & 130 & 130 \\
\hline \# pixels in $\mathrm{x}$ and $\mathrm{y}$ & 64 & 256 & 256 & $256 / 128$ & 256 \\
\hline Readout architecture & $\begin{array}{l}\text { Frame based } \\
\text { Sequential RW }\end{array}$ & $\begin{array}{l}\text { Frame based } \\
\text { Sequential RW }\end{array}$ & $\begin{array}{l}\text { Frame based } \\
\text { Sequential RW }\end{array}$ & $\begin{array}{l}\text { Frame based } \\
\text { Continuous RW }\end{array}$ & Data driven/frame based \\
\hline $\begin{array}{l}\text { Charge summing and allocation } \\
\text { mode (CSM) }\end{array}$ & No & No & No & Yes & No \\
\hline \# thresholds & 1 & $\begin{array}{l}2 \text { (window } \\
\text { discriminator) }\end{array}$ & 1 & $\begin{array}{l}2 / 4 \text { Seq RW } 1 / 4 \text { Cont } \\
\text { RW }\end{array}$ & 1 \\
\hline ToT/ToA & No & No & $\begin{array}{l}\text { ToT ( } 14 \text { bit) OR ToA } \\
\text { (14 bit, } 10 \mathrm{~ns} \\
\text { precision) }\end{array}$ & No & $\begin{array}{l}\text { ToT (10 bit) AND ToA (18 bit, } \\
1.56 \text { ns precision) }\end{array}$ \\
\hline Front end noise ( $\left.\mathrm{e}^{-} \mathrm{rms}\right)$ & 170 & 110 & 100 & $80(\mathrm{SPM}) 174(\mathrm{CSM})$ & 62 \\
\hline Peaking time (ns) & 100 & 150 & 100 & 120 & 30 \\
\hline Max count rate $\left(\mathrm{Mc} / \mathrm{mm}^{2} / \mathrm{s}\right)^{\mathrm{a}}$ & - & 826 & - & $\begin{array}{l}826(\text { SPM } 55 \mu \mathrm{m}) 164 \\
(\mathrm{CSM} 55 \mu \mathrm{m}) 376 \\
(\mathrm{SPM} 110 \mu \mathrm{m}) 28 \\
(\mathrm{CSM} 110 \mu \mathrm{m})\end{array}$ & 0.43 (data driven) \\
\hline $\begin{array}{l}\text { Number of sides available for } \\
\text { tiling }\end{array}$ & 0 & 3 & 3 & 3 & 3 \\
\hline
\end{tabular}

a Depends strongly on exact conditions of threshold, sensor material and energy of illumination.

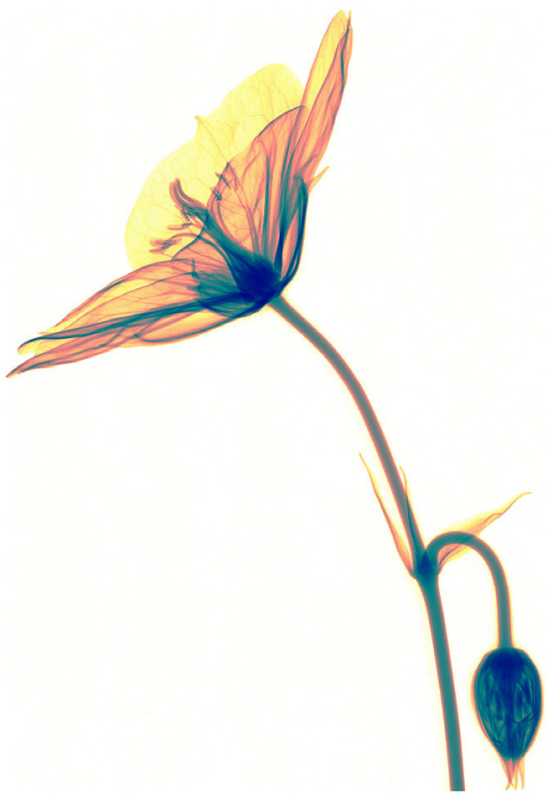

Fig. 15. Tiled X-ray image of a wild flower taken using the Medipix3RX chip connected to a $300 \mu \mathrm{m}$ thick silicon sensor (courtesy of S. Procz, University of Freiburg, Germany).

measure the angular correlation function between the two emitted $\mathrm{x}$ rays for the very first time [106]. Such experiments can be used to test atomic physics models.

Finally, it is also possible to use X-ray imaging detectors for nonscientific purposes. Fig. 15 is an X-ray image of a wild flower taken using an X-ray machine, and tiling Medipix3RX data. Science meets art.

\section{Summary, conclusions and future directions}

Three generations of ASICs using smaller and smaller feature sizes have been described and Table 1 summarises their main characteristics. Initially the higher component density was used to reduce the pixel size but more recently the aim has been to improve the functionality of a pixel. Two distinctive lines of development have been established. With the Medipix chips the aim has evolved from simple single photon counting towards spectroscopic X-ray imaging at relatively high rates.
Reasonable spectroscopic fidelity can only be preserved at high rates by using inter-pixel processing to mitigate the dispersive effects of charge sharing resulting from the charge deposition and collection mechanisms. The Timepix family of chips on the other hand seeks to transmit as much information as possible off-chip providing scope for many experiments where single charged particles have to be detected. A common feature has been the organic growth in the applications to which the chips have been applied thanks to a growing and active community using the chips and their suggestions for improvements. A particularly important contribution to this process came from the development of USB-based readout systems and software making the devices easily accessible and transportable.

Looking ahead we aim to further develop both families. For Medipix4 we aim to improve energy resolution at the same time as increasing the high flux capability. For Timepix 4 improved time resolution and possibly decreased pixel size are targeted. In both cases larger die sizes may be useful and we also aim to make use of Through Silicon Vias, whose feasibility has already been proven with Medipix3/Timepix3 processing [107], to provide chips which can be abutted on 4-sides.

In High Energy Physics, there is currently a strong interest in Monolithic Active Pixel sensors where sensor and readout electronics are integrated on the same substrate. These have the advantages that bump bonding is no longer required and that operation at even lower threshold levels $\left(\sim 100 \mathrm{e}^{-}\right)$becomes possible because of further reduced input capacitance and therefore noise. As a consequence, the sensors can be extremely thin and therefore lower mass. Doubtless these detectors will start to replace tracking layers in the large experiments initially on the border between the hybrid pixel layers and the strip detector layers. However, the advantages of the hybrid approach remain that almost any CMOS foundry can be used (minimising the risk of dependence on a single supplier), more components can be crammed into a pixel permitting operation in the hottest areas of high luminosity experiments, and the flexibility to combine the CMOS chip with almost any kind of detection medium.

\section{Acknowledgements}

The developments reported in this paper were made possible by the continued and strong support (intellectual, moral and material) of the members of the Medipix, Medipix2 and Medipix3 Collaborations. If a few groups are to be singled out for particular thanks the Institute for Experimental and Applied Physics at the Czech Technical University in Prague has contributed enormously to our efforts by providing and supporting the USB-based readout hardware and software. We also 
thank Nikhef for their long support with readout systems most recently for Timepix 3 and we do not forget the groups from Pisa and Naples who provided the same support in the early days. We thank all of those who have contributed to the ASIC design effort and in particular to Winnie Wong and Tuomas Poikela from CERN and Vladimir Gromov from Nikhef. We are also very grateful for the long-term support and guidance of our 'in house' detector experts, Erik Heijne and Lukas Tlustos. Jerome Alozy has provided crucial technical support both to the CERN designers and Collaboration members. Our thanks also go to the CERN KT group who have helped hugely in structuring our Collaborations and with licencing our chips to industry. PANalytical has been a long-term licensee and their continued contribution is also acknowledged.

\section{References}

[1] E.H.M. Heijne, Semiconductor micropattern pixel detectors: a review of the beginnings, Nucl. Instrum. Methods Phys. Res. A 465 (2001) 1-26.

[2] E.H.M. Heijne, F. Antinori, D. Barberis, K.H. Becks, H. Beker, W. Beusch, P. Burger, M. Campbell, E. Cantatore, M.G. Catanesi, E. Chesi, G. Darbo, S. D'Auria, C. DaVia', D. Di Bari, S. Di Liberto, T. Gys, G. Humpston, A. Jacholkowski, J.J. Jaeger, J. Jakubek, P. Jarron, W. Klempt, F. Krummenacher, K. Knudson, J. Kubasta, J.C. Lassalle, R. Leitner, F. Lemeilleur, V. Lenti, M. Letheren, B. Lisowski, L. Lopez, D. Loukas, M. Luptak, P. Martinengo, G. Meddeler, F. Meddi, P. Middelkamp, M. Morando, P. Morettini, A. Munns, P. Musico, F. Pellegrini, F. Pengg, S. Pospisil, E. Quercigh, J. Ridky, L. Rossi, K. Safarik, L. Scharfetter, G. Segato, S. Simone, K. Smith, W. Snoeys, C. Sobczynski, J. Stastny, V. Vrba, LHC1: A semiconductor pixel detector readout chip with internal, tunable delay providing a binary pattern of selected events, Nucl. Instrum. Methods Phys. Res. A 383 (1996) 55-61. http: //dx.doi.org/10.1016/S0168-9002(96)00658-4.

[3] C. Da Via, R. Bates, E. Bertolucci, U. Bottigli, M. Campbell, E. Chesi, M. Conti, S.D. Auria, C. Delpapa, M.E. Fantacci, G. Grossi, E. Heijne, E. Mancini, P. Middelkamp, C. Raine, P. Russo, V.O. Shea, L. Scharfetter, K. Smith, W. Snoeys, A. Stefanini, Gallium arsenide pixel detectors for medical imaging, Nucl. Instrum. Methods Phys. Res. A 395 (1997) 148-151.

[4] S.R. Amendolia, R. Beccherle, E. Bertolucci, M.G. Bisogni, U. Bottigli, E. Chesi, M.A. Ciocci, M. Conti, C. Da Via, A. Del Guerra, S. D’Auria, M.E. Fantacci, M. Gambaccini, G. Grossi, E. Heijnes, E. Mancini, R. Marchesini, P. Middelkamps, V.O. Shea, P. Randaccio, N. Romeo, V. Rosso, P. Russo, L. Scharfetter, K. Smith, W. Snoeys, A. Stefanini, Use of silicon and GaAs pixel detectors for digital autoradiography, IEEE Trans. Nucl. Sci. 44 (1997) 929-933.

[5] M. Campbell, E.H.M. Heijne, G. Meddeler, E. Pernigotti, W. Snoeys, A readout chip for a 64 x 64 pixel matrix with 15-bit single Photon Counting, IEEE Trans. Nucl. Sci. 45 (1998) 751-753.

[6] B. Mikulec, Single Photon Detection with Semiconductor Pixel Arrays for Medical Imaging Applications, CERN-THESIS-2000-021, 2000.

[7] P. Horowitz, W. Hill, The Art of Electronics, Cambrideg University Press, 1980

[8] P. Fischer, J. Hausmann, M. Overdick, B. Raith, N. Wermes, L. Blanquart, V. Bonzom, P. Delpierre, A counting pixel readout chip for imaging applications, Nucl. Instrum. Methods Phys. Res. A 405 (1998) 53-59. http://dx.doi.org/10. 1016/S0168-9002(97)01146-7.

[9] S.R. Amendolia, E. Bertolucci, M.G. Bisogni, U. Bottigli, M.A. Ciocci, M. Conti, P. Delogu, M.E. Fantacci, P. Maestro, V. Marzulli, E. Pernigotti, N. Romeo, V. Rosso, P. Russo, A. Stefanini, S. Stumbo, GaAs detector optimization for di ! erent medical imaging applications, Nucl. Instrum. Methods Phys. Res. A 434 (1999) 14-17.

[10] S. Manolopoulos, R. Bates, M. Campbell, W. Snoeys, E. Heijne, E. Pernigotti, C. Raine, K. Smith, J. Watt, V. O'Shea, J. Ludwig, C. Schwarz, X-ray imaging with photon counting hybrid semiconductor pixel detectors, Nucl. Instrum. Methods Phys. Res. A 434 (1999) 38-43. http://dx.doi.org/10.1016/S0168-9002(99)00430-1.

[11] L. Abate, E. Bertolucci, M. Conti, A. Di Cosmo, C. Di Cristo, G. Mettivier, M.C. Montesi, P. Russo, S. Fisiche, N. Federico, D. Zoologia, N. Federico, Quantitative dynamic imaging of biological processes with solid state radiation detector, IEEE Trans. Nucl. Sci. 47 (2000) 1907-1910.

[12] S.R. Amendolia, M.G. Bisogni, P. Delogu, M.E. Fantacci, G. Paternoster, V. Rosso, A. Stefanini, Characterization of a mammographic system based on single photon counting pixel arrays coupled to GaAs x-ray detectors, Med. Phys. 36 (2009) 13301339. http://dx.doi.org/10.1118/1.3097284.

[13] S.R. Amendolia, M.G. Bisogni, U. Bottigli, M.A. Ciocci, P. Delogu, G. Dipasquale, M.E. Fantacci, P. Maestro, V. Marzulli, B. Mikulec, E. Pernigotti, V. Rosso, A. Stefanini, S. Stumbo, Test of a GaAs-based pixel device for digital mammography, Nucl. Instrum. Methods Phys. Res. A 460 (2001) 50-54. http://dx.doi.org/10. 1016/S0168-9002(00)01095-0.

[14] C. Schwarz, M. Campbell, R. Goeppert, J. Ludwig, B. Mikulec, K. Runge, K.M. Smith, W. Snoeys, Measurements with Si and GaAs pixel detectors bonded to photon counting readout chips, Nucl. Instrum. Methods Phys. Res. A 466 (2001) 87-94. http://dx.doi.org/10.1016/S0168-9002(01)00829-4.
[15] X. Llopart, M. Campbell, R. Dinapoli, D. San Segundo, E. Pernigotti, Medipix2: A 64-k pixel readout chip with 55-um square elements working in single photon counting mode, IEEE Trans. Nucl. Sci. 49 (I) (2002) 2279-2283. http://dx.doi.org/ 10.1109/TNS.2002.803788.

[16] X. LLopart Cudié, Design and characterization of $64 \mathrm{~K}$ pixels chips working in single photon processing mode, CERN-THESIS-2007-062, 2007.

[17] F. Krummenacher, Pixel detectors with local intelligence: an IC designer point of view, Nucl. Instrum. Methods Phys. Res. A 305 (1991) 527-532. http://dx.doi.org/ 10.1016/0168-9002(91)90152-G.

[18] M. Campbell, 10 years of the Medipix2 collaboration, Nucl. Instrum. Methods Phys. Res. A 633 (2011) S1-S10. http://dx.doi.org/10.1016/j.nima.2010.06.106.

[19] M. Chmeissani, C. Frojdh, O. Gal, X. Llopart, J. Ludwig, M. Maiorino, E. Manach, G. Mettivier, M.C. Montesi, C. Ponchut, P. Russo, L. Tlustos, A. Zwerger, First experimental tests with a CdTe photon counting pixel detector hybridized with a Medipix2 readout chip, IEEE Trans. Nucl. Sci. 51 (2004) 2379-2385. http: //dx.doi.org/10.1109/TNS.2004.832324.

[20] M. Chmeissani, B. Mikulec, Performance limits of a single photon counting pixel system, Nucl. Instrum. Methods Phys. Res. A 460 (2001) 81-90.

[21] M. Chmeissani, M. Maiorino, G. Blanchot, G. Pellegrini, J. Garcia, M. Lozano, R. Martinez, C. Puigdengoles, M. Ullan, Charge sharing measurements of pixilated CdTe using medipix-II chip, in: Conf. Rec. - IEEE Instrum. Meas. Technol. Conf, Vol. 1, 2004, pp. 787-791. http://dx.doi.org/10.1109/IMTC.2004.1351164.

[22] L. Tlustos, Dissertation Performance and limitations of high granularity single photon processing X-ray imaging detectors, CERN-THESIS-2005-032, 2005.

[23] R. Ballabriga, M. Campbell, C. Frojdh, H.M. Heijne, X. Llopart, H.-E. Nilsson, L. Tlustos, Method for determining a particle and sensor device therefor, US Patent No. 7,667,205 B2, 2010.

[24] R. Ballabriga, M. Campbell, E.H.M. Heijne, X. Llopart, L. Tlustos, The Medipix3 prototype, a pixel readout chip working in single photon counting mode with improved spectrometric performance, in: 2006 IEEE Nucl. Sci. Symp. Conf. Rec., Vol. 54, 2006, pp. 3557-3561 http://dx.doi.org/10.1109/NSSMIC.2006.353767.

[25] R. Ballabriga Suné, The Design and Implementation in 0.13 micrometer CMOS of an Algorithm Permitting Spectroscopic Imaging with High Spatial Resolution for Hybrid Pixel Detectors, CERN-THESIS-2010-055, 2010.

[26] R. Ballabriga, M. Campbell, E. Heijne, X. Llopart, L. Tlustos, W. Wong, Medipix3: A $64 \mathrm{k}$ pixel detector readout chip working in single photon counting mode with improved spectrometric performance, Nucl. Instrum. Methods Phys. Res. A 633 (2011) S15-S18. http://dx.doi.org/10.1016/j.nima.2010.06.108.

[27] W. Wong, R. Ballabriga, M. Campbell, X. Llopart, L. Tlustos, Counter architectures for a single photon-counting pixel detector such as Medipix3, AIP Conf. Proc. 958 (2007) 262. http://dx.doi.org/10.1063/1.2825808.

[28] E.N. Gimenez, R. Ballabriga, M. Campbell, I. Horswell, X. Llopart, J. Marchal, K.J.S Sawhney, N. Tartoni, D. Turecek, Characterization of Medipix3 with synchrotron radiation, IEEE Trans. Nucl. Sci. 58 (2011) 323-332. http://dx.doi.org/10.1109/ TNS.2010.2089062.

[29] R. Ballabriga, J. Alozy, G. Blaj, M. Campbell, M. Fiederle, E. Frojdh, E.H.M. Heijne, X. Llopart, M. Pichotka, S. Procz, L. Tlustos, W. Wong, The Medipix3RX: a high resolution, zero dead-time pixel detector readout chip allowing spectroscopic imaging, J. Instrum. 8 (2013) C02016. http://dx.doi.org/10.1088/1748-0221/8/ 02/C02016.

[30] D. Pennicard, R. Ballabriga, X. Llopart, M. Campbell, H. Graafsma, Simulations of charge summing and threshold dispersion effects in Medipix3, Nucl. Instrum. Methods Phys. Res. A 636 (2011) 74-81. http://dx.doi.org/10.1016/j.nima.2011. 01.124.

[31] T. Koenig, E. Hamann, S. Procz, R. Ballabriga, A. Cecilia, M. Zuber, X. Llopart, M. Campbell, A. Fauler, T. Baumbach, M. Fiederle, Charge summing in spectroscopic X-ray detectors with high-Z sensors, IEEE Trans. Nucl. Sci. 60 (2013) 4713-4718. http://dx.doi.org/10.1109/TNS.2013.2286672.

[32] R. Ballabriga, J. Alozy, M. Campbell, E. Frojdh, E.H.M. Heijne, T. Koenig, X. Llopart, J. Marchal, D. Pennicard, T. Poikela, L. Tlustos, P. Valerio, W. Wong, M. Zuber, Review of hybrid pixel detector readout ASICs for spectroscopic Xray imaging, J. Instrum. 11 (2016). http://dx.doi.org/10.1088/1748-0221/11/01/ P01007. P01007-P01007.

[33] P. Colas, A.P. Colijn, A. Fornaini, Y. Giomataris, H. Van Der Graaf, E.H.M. Heijne, X. Llopart, J. Schmitz, J. Timmermans, J.L. Visschers, The readout of a GEM or Micromegas-equipped TPC by means of the Medipix2 CMOS sensor as direct anode, Nucl. Instrum. Methods Phys. Res. A 535 (2004) 506-510. http://dx.doi.org/10. 1016/j.nima.2004.07.180.

[34] X. Llopart, R. Ballabriga, M. Campbell, L. Tlustos, W. Wong, Timepix, a 65k programmable pixel readout chip for arrival time, energy and/or photon counting measurements, Nucl. Instrum. Methods Phys. Res. A 581 (2007) 485-494. http: //dx.doi.org/10.1016/j.nima.2007.08.079.

[35] T. Poikela, J. Plosila, T. Westerlund, M. Campbell, M. De Gaspari, X. Llopart, V. Gromov, R. Kluit, M. van Beuzekom, F. Zappon, V. Zivkovic, C. Brezina, K. Desch, Y. Fu, A. Kruth, Timepix3: a 65K channel hybrid pixel readout chip with simultaneous ToA/ToT and sparse readout, J. Instrum. 9 (2014) C05013. http: //dx.doi.org/10.1088/1748-0221/9/05/C05013.

[36] T. Poikela, Readout Architecture for Hybrid Pixel Readout Chips, CERN-THESIS2015-111, 2015. 
[37] M. De Gaspari, J. Alozy, R. Ballabriga, M. Campbell, E. Frojdh, J. Idarraga, S. Kulis, X. Llopart, T. Poikela, P. Valerio, W. Wong, Design of the analog front-end for the Timepix3 and Smallpix hybrid pixel detectors in $130 \mathrm{~nm}$ CMOS technology, J. Instrum. 9 (2014) C01037. http://dx.doi.org/10.1088/1748-0221/9/01/C01037.

[38] A. Kruth, C. Brezina, S. Celik, V. Gromov, R. Kluit, F. Zappon, K. Desch, H. Van Der Graaf, GOSSIPO-3: measurements on the prototype of a read-out pixel chip for Micro-Pattern Gaseous Detectors, J. Instrum. 5 (2010) C12005. http://dx.doi.org/ 10.1088/1748-0221/5/12/C12005.

[39] M. Campbell, X. Llopart Cudie, L. Tlustos, W.S.-W. Wong, R. Ballabriga Sune, G. Anton, T. Michel, M. Böhnel, K.L. Schwartz, U. Mollenhauer, et al. Radiation monitoring device, 2009, EP20070114122. http://www.google.com/patents/ EP2028509A1? $\mathrm{cl}=$ und.

[40] W.S. Wong, G. Anton, R. Ballabriga, M. Böhnel, M. Campbell, E. Heijne, X. Llopart, T. Michel, I. Münster, R. Plackett, P. Sievers, P. Takoukam, L. Tlustos, P. Valerio, A pixel detector asic for dosimetry using time-over-threshold energy measurements, Radiat. Meas. 46 (2011) 1619-1623. http://dx.doi.org/10.1016/j.radmeas.2011. 06.061.

[41] W.S. Wong, G. Anton, R. Ballabriga, G. Blaj, M. Böhnel, M. Campbell, T. Gabor, E. Heijne, X. Llopart, T. Michel, I. Ritter, T. Poikela, P. Sievers, L. Tlustos, P. Valerio, Electrical measurements of a multi-mode hybrid pixel detector ASIC for radiation detection, J. Instrum. 7 (2012) C01056. http://dx.doi.org/10.1088/1748-0221/7/ 01/C01056.

[42] W.S. Wong, A Hybrid Pixel Detector ASIC with Energy Binning for Real-Time, Spectroscopic Dose Measurements, Mid Sweden University Doctoral Thesis 128, 2012.

[43] K. Akiba, M. Artuso, R. Badman, A. Borgia, R. Bates, F. Bayer, M. Van Beuzekom, J. Buytaert, E. Cabruja, M. Campbell, P. Collins, M. Crossley, R. Dumps, L. Eklund, D. Esperante, C. Fleta, A. Gallas, M. Gandelman, J. Garofoli, M. Gersabeck, V.V. Gligorov, H. Gordon, E.H.M. Heijne, V. Heijne, D. Hynds, M. John, A. Leflat, L. Ferre Llin, X. Llopart, M. Lozano, D. Maneuski, T. Michel, M. Nicol, M. Needham, C. Parkes, G. Pellegrini, R. Plackett, T. Poikela, E. Rodrigues, G. Stewart, J. Wang, Z. Xing, Charged particle tracking with the Timepix ASIC, Nucl. Instrum. Methods Phys. Res. A 661 (2012) 31-49. http://dx.doi.org/10.1016/j.nima.2011.09.021.

[44] P. Collins, R. Linder, LHCb VELO Upgrade Techgnical Design Report, 2013.

[45] T. Poikela, M. De Gaspari, J. Plosila, T. Westerlund, R. Ballabriga, J. Buytaert, M. Campbell, X. Llopart, K. Wyllie, V. Gromov, M. van Beuzekom, V. Zivkovic, VeloPix: the pixel ASIC for the LHCb upgrade, J. Instrum. 10 (2015) C01057. http://dx.doi.org/10.1088/1748-0221/10/01/C01057.

[46] L. Linssen, A. Miyamoto, M. Stanitzki, H. Weerts, Physics and Detectors at CLIC: CLIC Conceptual Design Report., 2012. http://dx.doi.org/10.5170/CERN-2012003.

[47] P. Valerio, J. Alozy, S. Arfaoui, R. Ballabriga, M. Benoit, S. Bonacini, M. Campbell, D. Dannheim, M. De Gaspari, D. Felici, S. Kulis, X. Llopart, A. Nascetti, T. Poikela, W.S. Wong, A prototype hybrid pixel detector ASIC for the CLIC experiment, J. Instrum. 9 (2014) C01012. http://dx.doi.org/10.1088/1748-0221/9/01/C01012.

[48] Z. Vykydal, J. Jakubek, S. Pospisil, USB interface for Medipix2 pixel device enabling energy and position-sensitive detection of heavy charged particles, Nucl. Instrum. Methods Phys. Res. A 563 (2006) 112-115. http://dx.doi.org/10.1016/j.nima. 2006.01.114.

[49] V. Kraus, M. Holik, M. Platkevic, J. Jakubek, J. Vallerga, R. Raffanti, M. Kroupa, J. Jakubek, P. Soukup, M. Holik, V. Kraus, P. Masek, V. Linhart, FITPix — fast interface for Timepix pixel detectors, J. Instrum. 6 (2011) C01079. http://dx.doi. org/10.1088/1748-0221/6/01/C01079.

[50] Z. Vykydal, J. Jakubek, Nuclear instruments and methods in physics research A USB Lite - miniaturized readout interface for Medipix2 detector, Nucl. Instrum. Methods Phys. Res. A 633 (2011) S48-S49. http://dx.doi.org/10.1016/j.nima. 2010.06.118.

[51] D. Tureček, T. Holy, J. Jakubek, S. Pospisil, Z. Vykydal, Pixelman: a multiplatform data acquisition and processing software package for Medipix2, Timepix and Medipix3 detectors, J. Instrum. 6 (2011) C01046. http://dx.doi.org/10.1088/ 1748-0221/6/01/C01046.

[52] T. Whyntie, M.A. Harrison, Simulation and analysis of the LUCID experiment in the Low Earth Orbit radiation environment, J. Phys: Conf. Ser. 513 (2014). http://dx.doi.org/10.1088/1742-6596/513/2/022038.

[53] N. Stoffle, L. Pinsky, M. Kroupa, S. Hoang, J. Idarraga, C. Amberboy, R. Rios, J. Hauss, J. Keller, A. Bahadori, E. Semones, D. Turecek, J. Jakubek, Z. Vykydal, S. Pospisil, Timepix-based radiation environment monitor measurements aboard the International Space Station, Nucl. Instrum. Methods Phys. Res. A 782 (2015) 143-148. http://dx.doi.org/10.1016/j.nima.2015.02.016.

[54] M. Kroupa, A. Bahadori, T. Campbell-ricketts, A. Empl, S. Minh, J. Idarraga-munoz, R. Rios, E. Semones, N. Stoffle, L. Tlustos, D. Turecek, L. Pinsky, A semiconductor radiation imaging pixel detector for space radiation dosimetry, Life Sci. Sp. Res. 6 (2015) 69-78. http://dx.doi.org/10.1016/j.lssr.2015.06.006.

[55] S. Gohl, B. Bergmann, C. Granja, A. Owens, M. Pichotka, S. Pospisil, Measurement of particle directions in low earth orbit with a Timepix, J. Instrum. 11 (2016) C11023. http://dx.doi.org/10.1088/1748-0221/11/11/C11023.

[56] C. Granja, S. Polansky, Z. Vykydal, S. Pospisil, A. Owens, Z. Kozacek, K. Mellab, M. Simcak, The SATRAM Timepix spacecraft payload in open space on board the Proba-V satellite for wide range radiation monitoring in LEO orbit, Planet. Space Sci. 125 (2016) 114-129.
[57] C. Ponchut, J. Clément, J.M. Rigal, E. Papillon, J. Vallerga, D. LaMarra, B. Mikulec, Photon-counting X-ray imaging at kilohertz frame rates, Nucl. Instrum. Methods Phys. Res. A 576 (2007) 109-112. http://dx.doi.org/10.1016/j.nima.2007.01.131.

[58] C. Ponchut, J.M. Rigal, J. Clément, E. Papillon, A. Homs, S. Petitdemange, MAXIPIX, a fast readout photon-counting X-ray area detector for synchrotron applications, J. Instrum. 6 (2011) C01069. http://dx.doi.org/10.1088/1748-0221/ 6/01/C01069.

[59] S. Huotari, C.J. Sahle, C. Henriquet, K. Martel, L. Simonelli, R. Verbeni, H, Gonzalez, M. Lagier, C. Ponchut, M. Moretti Sala, M. Krisch, G. Monaco, A large-solid-angle X-ray Raman scattering spectrometer at ID20 of the European Synchrotron Radiation Facility, J. Synchrotron Radiat. 24 (2017) 521-530.

[60] D. Pennicard, S. Smoljanin, B. Struth, H. Hirsemann, A. Fauler, M. Fiederle, O. Tolbanov, A. Zarubin, A. Tyazhev, G. Shelkov, H. Graafsma, The LAMBDA photoncounting pixel detector and high-Z sensor development, J. Instrum. 9 (2014) C12026. http://dx.doi.org/10.1088/1748-0221/9/12/C12026.

[61] R. Plackett, I. Horswell, E.N. Gimenez, J. Marchal, I. Horswell, D. Omar, N. Tartoni, Merlin: a fast versatile readout system for Medipix3, J. Instrum. 8 (2013) C01038. http://dx.doi.org/10.1088/1748-0221/8/01/C01038.

[62] G. Crevatin, I. Horswell, D. Omar, N. Tartoni, S. Carrato, G. Caotero, Development of a Timepix3 readout system based on the Merlin readout system, J. Instrum. 10 (2015) C03042. http://dx.doi.org/10.1088/1748-0221/10/03/C03042.

[63] J. Marchal, I. Horswell, B. Willis, R. Plackett, E.N. Gimenez, J. Spiers, D. Ballard, P. Booker, J.A. Thompson, P. Gibbons, S.R. Burge, T. Nicholls, J. Lipp, N. Tartoni, EXCALIBUR : a small-pixel photon counting area detector for coherent X-ray diffraction - Front-end design, fabrication and characterisation, J. Phys: Conf. Ser. 425 (2013). http://dx.doi.org/10.1088/1742-6596/425/6/062003.

[64] S. Redford, CLIC vertex detector R\&D, J. Instrum. 9 (2014) C08014. http://dx.doi. org/10.1088/1748-0221/9/08/C08014.

[65] C. Fröjdh, H. Graafsma, H.E. Nilsson, C. Ponchut, Characterization of a pixellated CdTe detector with single-photon processing readout, Nucl. Instrum. Methods Phys. Res. A 563 (2006) 128-132. http://dx.doi.org/10.1016/j.nima.2006.01.076.

[66] P. Cermak, I. Stekl, V. Bocarov, J. Jakubek, S. Pospisil, M. Fiederle, K. Zuber, The characterization of CdTe TimePix device and the study of its capabilities for the double beta decay measurements, in: IEEE Nucl. Sci. Symp. Conf. Rec., 2008, pp. 444-445.

[67] M. Platkevic, P. Cermak, J. Jakubek, S. Pospisil, I. Stekl, Z. Vykydal, J. Zemlicka, C. Leroy, P. Allard, G. Bergeron, P. Soueid, C. Teyssier, R. Yapoudjian, M. Fiederle, A. Fauler, G.A. Chelkov, O. Tolbanov, A. Tyazhev, J. Vissers, Characterization of charge collection in various semiconductor sensors with energetic protons and Timepix device, in: IEEE Nucl. Sci. Symp. Conf. Rec., 2012, pp. 4715-4719 http: //dx.doi.org/10.1109/NSSMIC.2011.6154765.

[68] E. Hamann, T. Koenig, M. Zuber, A. Cecilia, A. Tyazhev, O. Tolbanov, S. Procz, A. Fauler, T. Baumbach, M. Fiederle, Performance of a Medipix3RX spectroscopic pixel detector with a high resistivity gallium arsenide sensor, IEEE Trans. Med. Imaging 34 (2015) 707-715. http://dx.doi.org/10.1109/TMI.2014.2317314.

[69] M. Zuber, E. Hamann, R. Ballabriga, M. Campbell, M. Fiederle, T. Baumbach, T. Koenig, An investigation into the temporal stability of CdTe-based photon counting detectors during spectral micro-CT acquisitions, Biomed. Phys. Eng. Express 1 (2015) 25205. http://dx.doi.org/10.1088/2057-1976/1/2/025205.

[70] J. Jakubek, Energy-sensitive X-ray radiography and charge sharing effect in pixelated detector, Nucl. Instrum. Methods Phys. Res. A 607 (2009) 192-195. http://dx.doi.org/10.1016/j.nima.2009.03.148.

[71] L. Abate, E. Bertolucci, M. Conti, M.C. Montesi, P. Russo, GaAs pixel arrays for beta imaging in medicine and biology, Nucl. Instr. Meth. A 460 (2001) 97-106. http://dx.doi.org/10.1016/S0168-9002(00)01102-5.

[72] P. Russo, A. Lauria, M. Marotta, G. Mettivier, M.C. Montesi, L. Aloji, S. Lastoria, ${ }^{18}$ F-FDG positron autoradiography with a particle counting silicon pixel detector, Phys. Med. Biol. 53 (2008) 6227-6243. http://dx.doi.org/10.1088/0031-9155/ 53/21/022.

[73] M. Esposito, J. Jakubek, G. Mettivier, S. Pospisil, P. Russo, J. Solc, Energy sensitive Timepix silicon detector for electron imaging, Nucl. Instr. Meth. A 652 (2011) 458461. http://dx.doi.org/10.1016/j.nima.2011.01.148.

[74] M. Esposito, G. Mettivier, P. Russo, C-14 autoradiography with an Energy sensitive silicon pixel detector, Phys. Med. Biol. 56 (2011) 1947-1965. http://dx.doi.org/ 10.1088/0031-9155/56/7/003.

[75] J. Jakubek, G. Mettivier, M.C. Montesi, S. Pospisil, P. Russo, CdTe hybrid pixel detector for imaging with thermal neutrons, Nucl. Instr. Meth. A 563 (2006) 238241. http://dx.doi.org/10.1016/j.nima.2006.01.134.

[76] J. Dudak, J. Zemlicka, J. Karch, M. Patzelt, J. Mrzilkova, P. Zach, Z. Hermanova, J. Kvacek, F. Krejci, High-contrast X-ray micro- radiography and micro-CT of ex-vivo soft tissue murine organs utilizing ethanol fixation and large area photon-counting detector, Sci. Rep. 6 (2016) 1-9. http://dx.doi.org/10.1038/srep30385.

[77] T. Koenig, M. Zuber, E. Hamann, A. Cecilia, R. Ballabriga, M. Campbell, M. Ruat, L. Tlustos, A. Fauler, M. Fiederle, T. Baumbach, How spectroscopic x-ray imaging benefits from inter-pixel communication, Phys. Med. Biol. 59 (2014) 6195-6213. http://dx.doi.org/10.1088/0031-9155/59/20/6195.

[78] S. Procz, K. Wartig, A. Fauler, A. Zwerger, J. Luebke, R. Ballabriga, G. Blaj, M. Campbell, M. Mix, M. Fiederle, Medipix3 CT for material sciences, J. Instrum. 8 (2013) C01025. http://dx.doi.org/10.1088/1748-0221/8/01/C01025. 
[79] M.F. Walsh, a.M.T. Opie, J.P. Ronaldson, R.M.N. Doesburg, S.J. Nik, J.L. Mohr, R. Ballabriga, a.P.H. Butler, P.H. Butler, First CT using Medipix3 and the MARS-CT-3 spectral scanner, J. Instrum. 6 (2011) C01095. http://dx.doi.org/10.1088/17480221/6/01/C01095.

[80] J. Idarraga, et al. Towards energy-resolved mammography. Phys. Med. Biol. (in preparation) (n.d.).

[81] R. Aamir, et al., MARS spectral molecular imaging of lamb tissue: data collection and image analysis, J. Instrum. 9 (2014) P02005. http://dx.doi.org/10.1088/17480221/9/02/P02005.

[82] A.R. Faruqi, D.M. Cattermole, R. Henderson, B. Mikulec, C. Raeburn, Evaluation of a hybrid pixel detector for electron microscopy, Ultramicroscopy 94 (2003) 263276.

[83] G. McMullan, A.R. Faruqi, Electron microscope imaging of single particles using the Medipix2 detector, Nucl. Instrum. Methods Phys. Res. A 591 (2008) 129-133. http://dx.doi.org/10.1016/j.nima.2008.03.041.

[84] R. Van Gastel, I. Sikharulidze, S. Schramm, J.P. Abrahams, B. Poelsema, R.M. Tromp, Ultramicroscopy Medipix 2 detector applied to low energy electron microscopy, Ultramicroscopy 110 (2009) 33-35. http://dx.doi.org/10.1016/j. ultramic.2009.09.002.

[85] G. Mcmullan, D.M. Cattermole, S. Chen, R. Henderson, X. Llopart, C. Summerfield, L. Tlustos, A.R. Faruqi, Electron imaging with Medipix2 hybrid pixel detector, Ultramicroscopy 107 (2007) 401-413. http://dx.doi.org/10.1016/j.ultramic.2006. 10.005 .

[86] M. Krajnak, D. Mcgrouther, D. Maneuski, V.O. Shea, S. Mcvitie, Pixelated detectors and improved efficiency for magnetic imaging in STEM differential phase contrast, Ultramicroscopy 165 (2016) 42-50. http://dx.doi.org/10.1016/j.ultramic.2016. 03.006.

[87] J. Kaminski, Y. Bilevych, K. Desch, C. Krieger, M. Lupberger, GridPix detectorsintroduction and applications, Nucl. Instrum. Methods Phys. Res. A 845 (2017) 233-235. http://dx.doi.org/10.1016/j.nima.2016.05.134.

[88] A.K. Biegun, J. Visser, T. Klaver, N. Ghazanfari, M.-J. van Giethem, E. Koffeman, M. Van Beuzekom, S. Brandenburg, Proton radiography with timepix based time projection chambers, IEEE Trans. Med. Imaging 35 (2016) 1099-1105.

[89] J. Vallerga, J. McPhate, A. Tremsin, O. Siegmund, High-resolution UV, alpha and neutron imaging with the Timepix CMOS readout, Nucl. Instrum. Methods Phys. Res. A 591 (2008) 151-154. http://dx.doi.org/10.1016/j.nima.2008.03.046.

[90] A.S. Tremsin, S.C. Vogel, M. Mocko, M.A. Bourke, V. Yuan, R.O. Nelson, D.W. Brown, W.B. Feller, Non-destructive studies of fuel rodlets by neutron resonance absorption radiography and thermal neutron radiography, J. Nucl. Mater. 440 (2013) 633-646.

[91] A.S. Tremsin, J. Rakovan, T. Shinohara, W. Kockelmann, A.S. Losko, S.C. Vogel, Non-destructive study of bulk crystallinity and elemental composition of natural gold single crystal samples by energy-resolved neutron imaging, Sci. Rep. (2017).

[92] A.S. Tremsin, J.V. Vallerga, J.B. Mcphate, O.H.W. Siegmund, Optimization of high count rate event counting detector with Microchannel Plates and quad Timepix readout, Nucl. Instrum. Methods Phys. Res. A 787 (2015) 20-25. http://dx.doi. org/10.1016/j.nima.2014.10.047.

[93] J.H. Jungmann, R.M.A. Heeren, Detection systems for mass spectrometry imaging : A perspective on novel developments with a focus on active pixel detectors, Rapid Commun. Mass Spectrom. 27 (2013) 1-23. http://dx.doi.org/10.1002/rcm.6418.

[94] N. Pacifico, S. Aghion, J. Alozy, C. Amsler, A. Ariga, T. Ariga, G. Bonomi, P. Braeunig, J. Bremer, R.S. Brusa, L. Cabaret, M. Caccia, M. Campbell, R. Caravita, F. Castelli, G. Cerchiari, K. Chlouba, S. Cialdi, D. Comparat, G. Consolati, A. Demetrio, L. Di Noto, M. Doser, A. Dudarev, A. Ereditato, C. Evans, R. Ferragut, J. Fesel, A. Fontana, S. Gerber, M. Giammarchi, A. Gligorova, F. Guatieri, S. Haider, H. Holmestad, T. Huse, E. Jordan, A. Kellerbauer, M. Kimura, D. Krasnicky, V. Lagomarsino, P. Lansonneur, G. Lawler, P. Lebrun, X. Llopart, C. Malbrunot, S. Mariazzi, L. Marx, V. Matveev, Z. Mazzotta, G. Nebbia, P. Nedelec, M. Oberthaler, D. Pagano, L. Penasa, V. Petracek, C. Pistillo, F. Prelz, M. Prevedelli, L. Ravelli, L. Resch, O.M. Roehne, A. Rotondi, M. Sacerdoti, H. Sandaker, R. Santoro, P. Scampoli, L. Smestad, F. Sorrentino, M. Spacek, J. Storey, I.M. Strojek, G. Testera, I. Tietje, L. Tlustos, E. Widmann, P. Yzombard, S. Zavatarelli, J. Zmeskal, N. Zurlo, Direct detection of antiprotons with the Timepix3 in a new electrostatic selection beamline, Nucl. Instrum. Methods Phys. Res. A 831 (2015) 12-17. http: //dx.doi.org/10.1016/j.nima.2016.03.057.
[95] S. Levasseur, B. Denning, S. Gibson, H. Sandberg, M. Sapinski, G. Schneider, J. Storey, Development of a rest gas ionisation profile monitor for the CERN Proton Synchrotron based on a Timepix3 pixel detector, J. Instrum. 12 (2017) C02050. http://dx.doi.org/10.1088/1748-0221/12/02/C02050.

[96] B. Bergmann, I. Caicedo, C. Leroy, S. Pospisil, Z. Vykydal, ATLAS-TPX: a two-layer pixel detector setup for neutron detection and radiation field characterization, J. Instrum. 11 (2016) P10002. http://dx.doi.org/10.1088/1748-0221/11/10/P10002.

[97] A. Sopczak, B. Ali, T. Asawatavonvanich, J. Begera, B. Bergmann, T. Billoud, P. Burian, I. Caicedo, D. Caforio, E. Heijne, J. Janecek, C. Leroy, P. Mánek, K. Mochizuki, Y. Mora, J. Pacík, C. Papadatos, M. Platkevi, S. Polansky, S. Pospíšil, M. Suk, Z. Svoboda, Precision luminosity of LHC proton-proton collisions at 13 TeV using hit counting with TPX pixel devices, IEEE Trans. Nucl. Sci. 64 (2017) 915-924.

[98] K. Gwosch, B. Hartmann, J. Jakubek, S. Pospisil, O. Jiikel, M. Martisikova, 3D Beam Monitoring for $12 \mathrm{C}$ Radiotherapy by Tracking of Secondary Ions U sing the Timepix Detector, in: IEEE Nucl. Sci. Symp. Med. Imaging Conf. Rec., 2012, pp. 1291-1294.

[99] L. Opalka, C. Granja, B. Hartmann, J. Jakubek, O. Jaekel, M. Martisikova, S. Pospisil, J. Solc, Linear energy transfer and track pattern recognition of secondary radiation generated in hadron therapy beam in a PMMA target, J. Instrum. 8 (2013) C02047. http://dx.doi.org/10.1088/1748-0221/8/02/C02047.

[100] S. Alnaghy, D.L. Cutajar, J.A. Bucci, K. Enari, M. Safavi-Naeini, M. Favoino, M. Tartaglia, F. Carriero, J. Jakubek, S. Pospisil, M. Lerch, A.B. Rosenfeld, M. Petasecca, BrachyView: Combining LDR seed positions with transrectal ultrasound imaging in a prostate gel phantom, Phys. Med. 34 (2017) 55-64. http://dx.doi.org/ 10.1016/j.ejmp.2017.01.012.

[101] J. Jakubek, M. Platkevic, C. Granja, U. Köster, S. Pospisil, Direct observation of decay of radioactive nuclei with spatial and time coincidence technique, Nucl. Instrum. Methods Phys. Res. A 633 (2011) S203-S205. http://dx.doi.org/10.1016/ j.nima.2010.06.167.

[102] B. Bergmann, S. Pospisil, S. Member, I. Caicedo, J. Kierstead, H. Takai, E. Frojdh, Ionizing energy depositions after fast neutron interactions in silicon, IEEE Trans. Nucl. Sci. 63 (2016) 2372-2378.

[103] D. Vavrik, J. Jakubek, S. Pospisil, J. Vacik, Position sensitive detection of neutrons in high radiation background field, Rev. Sci. Instrum. 85 (2014). http://dx.doi.org/ 10.1063/1.4862478.

[104] F. Krejci, J. Zemlicka, J. Jakubek, J. Dudak, D. Vavrik, U. Köster, D. Atkins, A. Kaestner, J. Soltes, L. Viererbl, J. Vacik, I. Tomandl, Development and characterization of high-resolution neutron pixel detectors based on Timepix read-out chips, J. Instrum. 11 (2016) C12026. http://dx.doi.org/10.1088/1748-0221/11/ 12/C12026.

[105] T. Michel, B. Bergmann, M. Filipenko, T. Gleixner, K. Zuber, Measurement of the double $\mathrm{K}$-shell vacancy creation probability in the electron-capture decay of 55 Fe with active-pixel detectors, Phys. Rev. C 89 (2014) 1-15. http://dx.doi.org/10. 1103/PhysRevC.89.014609.

[106] B. Bergmann, T. Michel, A. Surzhykov, S. Fritzsche, Angular correlation function of the hypersatellite-satellite X-ray cascade following K-shell electron capture of 55 Fe, Phys. Rev. C 94 (2016).

[107] M. Campbell, J. Alozy, R. Ballabriga, E. Frojdh, E. Heijne, X. Llopart, T. Poikela, L. Tlustos, P. Valerio, W. Wong, Towards a new generation of pixel detector readout chips, J. Instrum. 11 (2016) C01007. http://dx.doi.org/10.1088/17480221/11/01/C01007. 